\title{
Muon Energy Reconstruction Through the Multiple Scattering Method in the NO $\nu$ A Detectors
}

\author{
A THESIS \\ SUBMITTED TO THE FAGULTY OF THE GRADUATE SGHOOL \\ OF THE UNIVERSITY OF MINNESOTA \\ BY
}

Silvia Fernanda Psihas Olmedo

\begin{abstract}
IN PARTIAL FULFILLMENT OF THE REQUIREMENTS
FOR THE DEGREE OF

MASTER OF SGIENGE
\end{abstract}

ADVISOR: Dr. Alec Habig

June, 2013 
(C) Silvia Fernanda Psihas Olmedo 2013 ALL RIGHTS RESERVED 


\section{Acknowledgements}

Thanks to God, I had many people who helped, guided and inspired me through my first years in graduate school and to whom I will be forever grateful.

I owe my gratitude to my advisor, Dr. Alec Habig, who opened the door to what has been the greatest learning experience of my life: working in the $\mathrm{NO} \nu \mathrm{A}$ collaboration. His guidance taught me valuable lessons about physics and showed me what it is to be an ethical and thorough scientist. The lessons I learned from Dr. Habig will forever influence my views of science as a profession.

Besides my advisor, I would like to thank Dr. Richard Gran for his contribution to my work. There were many "quick" questions I asked Dr. Gran which turned into hours of insight and, later, weeks of inspiration.

This work wouldn't be possible without the $\mathrm{NO} \nu \mathrm{A}$ collaboration. I would like to thank everyone who welcomed me to $\mathrm{NO} \nu \mathrm{A}$ with helpful advice. Especially Dr. Mayly Sanchez and Dr. Jonathan Paley for their guidance through this work and beyond it.

I would not have made it this far without the support of my loving family. I want to thank my mother who has been my greatest friend and support through the years and my brother Angel who is my inspiration to pursue my dreams. I also thank my father and my close family whose belief in me is the fuel for my own.

I would like to thank my partner, Justin Vasel. Holding his hand throughout the way helped me turn doubt into success and struggle into adventure. 


\begin{abstract}
Neutrino energy measurements are a crucial component in the experimental study of neutrino oscillations. These measurements are done through the reconstruction of neutrino interactions and energy measurements of their products. This thesis presents the development of a technique to reconstruct the energy of muons from neutrino interactions in the $\mathrm{NO} \nu \mathrm{A}$ experiment. This is achieved through the understanding of muon multiple scattering within the $\mathrm{NO} \nu \mathrm{A}$ detectors. This technique is particularly useful for estimating energies of muons which escape the detector.
\end{abstract}




\section{Contents}

$\begin{array}{ll}\text { Acknowledgements } & \text { i }\end{array}$

$\begin{array}{ll}\text { Abstract } & \text { ii }\end{array}$

List of Figures $\quad$ v

1 Neutrinos and the NO $\nu$ A Experiment 1

1.1 Neutrino Oscillations . . . . . . . . . . . . . . . . 2

1.1.1 Oscillations Theory . . . . . . . . . . . . 2

1.1.2 Experimental Measurements ............. 5

1.2 The $\mathrm{NO} \nu \mathrm{A}$ Experiment . . . . . . . . . . . . . . . . . 7

1.2.1 Overview ..................... 7

1.2.2 The $\mathrm{NO} \nu \mathrm{A}$ Detectors . . . . . . . . . . . . . . . 8

1.2.3 $\nu_{\mu}$ Oscillation Measurements . . . . . . . . . . . . 10

2 Muons in the NO $\nu$ A Detectors $\quad 11$

2.1 Muon Behavior in the Detectors . . . . . . . . . . . . . . 11

2.2 Muon Energy Reconstruction . . . . . . . . . . . . . . . 13

2.2.1 Reconstruction Methodology . . . . . . . . . . . . 13

2.2.2 Reconstruction Method Evaluation . . . . . . . . . . . . 14

2.3 Escaping muons . . . . . . . . . . . . . . . . . . 15

2.4 Muon Multiple Scattering . . . . . . . . . . . . . . 16

2.4.1 Multiple Scattering and Energy Reconstruction . . . . . . . . . 18 
3 Scattering Angle Reconstruction $\quad 20$

3.1 Reconstructed Track Structure . . . . . . . . . . . . . . . . . 21

3.2 Extracting Scattering Angles from Tracks . . . . . . . . . . . . . . 22

3.3 Angle to Energy Correlations . . . . . . . . . . . . . . . . 24

3.3.1 Measurements using track segments . . . . . . . . . . 25

3.3.2 Gauging Multiple Scattering . . . . . . . . . . . . . 27

4 Muon Energy Reconstruction $\quad 29$

4.1 Single Variable Approach Methodology . . . . . . . . . . . . . . . . . 29

4.1.1 Single Variable Method Evaluation and Results . . . . . . . . . 32

4.2 Multi-Variate Approach Methodology . . . . . . . . . . . . . . 35

4.2.1 Multi-Variable Method Evaluation and Results . . . . . . . . . 36

5 Discussion of Results $\quad 39$

5.1 Statistical Impact of this Method . . . . . . . . . . . . . . . . . 40

5.2 Conclusion ........................... 42

$\begin{array}{ll}\text { References } & 43\end{array}$

Appendix A. The NO $\nu$ A Event Generator and the Single Muons Sample 44

A.1 Single Muon Production . . . . . . . . . . . . . . . . 46

A.2 NuMI beam simulations for $\mathrm{NO} \nu \mathrm{A} \ldots \ldots \ldots$. . . . . . . . 47

Appendix B. The use of Artificial Neural Network for Data Analysis 49

B.1 ROOT and the ANN functionality . . . . . . . . . . . . . . . 51

B.2 ANN testing . . . . . . . . . . . . . . . 53 


\section{List of Figures}

1.1 The Standard Model of Particle Physics . . . . . . . . . . . . . . . . 1

$1.2 \mathrm{NO} \nu \mathrm{A}$ Expected Sensitivity . . . . . . . . . . . . . . . . . 5

1.3 Neutrino interactions in the $\mathrm{NO} \nu \mathrm{A}$ detectors . . . . . . . . . . . . 6

1.4 Distance and Angle Design Constraints for the NO $\nu$ A Detectors . . . . 8

1.5 Single NO $\nu$ A Detector Element . . . . . . . . . . . . . . . . . 9

$1.6 \mathrm{NO} \nu \mathrm{A}$ Detector Geometry . . . . . . . . . . . . . . . . . . . . . 9

$1.7 \mathrm{NO} \nu \mathrm{A}$ Expected Oscillated Neutrino Signal . . . . . . . . . . . . . . . 10

2.1 Deposited Energy Distribution For Muons . . . . . . . . . . . . . . . 12

2.2 Sample Neutrino Interaction in the NO $\nu$ A Event Display . . . . . . . 12

2.3 Correlation Between Summed Deposited Energy and True Energy . . . 13

2.4 Correlation Between Muon Track Length and True Energy . . . . . . . 14

2.5 Evaluation of the Track Length Reconstruction Method . . . . . . . . . 15

2.6 Expected Muon Multiple Scattering Behavior in the NO $\nu$ A Detectors . 17

3.1 Small Scattering Impact on Tracks Diagram . . . . . . . . . . . . . . 21

3.2 Reconstructed Tracks in the NO $\nu$ A Event Display . . . . . . . . . . . . 21

3.3 Track Vector and Angle Measurement Diagram . . . . . . . . . . . . . 22

3.4 Track Vector and Angle Measurement Diagram . . . . . . . . . . . . . 23

3.5 Correlation Between angleSigma from Full Tracks and True Muon En-

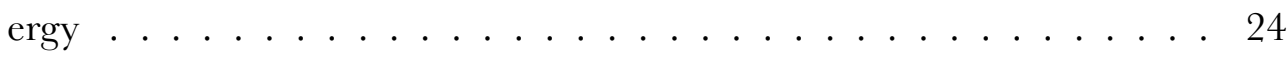

3.6 Correlation Between angleSigma from 4 meter segments and True Muon

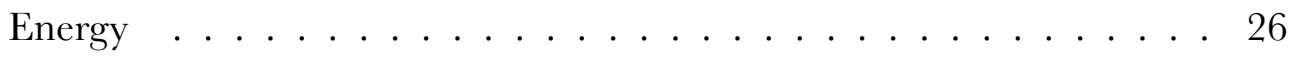

3.7 Correlation Between angleSigma and True Muon Energy (2, 3 and $4 \mathrm{~m}) 27$ 
3.8 Correlation Between angle Variables and True Muon Energy) . . . . . 28

4.1 Profiles with Function Fit of Correlations between Angle Variables and True Muon Energy . . . . . . . . . . . . . . . . . . 30

4.2 Evaluation of the Scattering Angle“"Table Lookup" Energy Reconstruction Method . . . . . . . . . . . . . . . . . . . . 33

4.3 Evaluation of the Multiple Scattering Energy Reconstruction Method on a Single Muon Sample . . . . . . . . . . . . . . . . 36

4.4 Evaluation of the Multiple Scattering Energy Reconstruction Method on NuMI MC . . . . . . . . . . . . . . . . . . . . 37

5.1 The NO $\nu$ A Expected Sensitivity with Uncontained Sample Contributions 40

5.2 Fraction of Uncontained Muons to be Impacted by the Multiple Scattering Method . . . . . . . . . . . . . . . . . . . 41

A.1 Characteristics of Muons from NuMI beam Neutrino Interactions _ . . 47

B.1 ANN Functionality Diagram . . . . . . . . . . . . . . . 50

B.2 ANN Monitoring Tools . . . . . . . . . . . . . . . . . 51

B.3 ANN Performance . . . . . . . . . . . . . . . . 52

B.4 Single Input ANN Tests . . . . . . . . . . . . . . . . . . . . 54 


\section{Chapter 1}

\section{Neutrinos and the NO $\nu$ A Experiment}

Particle physicists of our generation have the privilege of contributing to one of the most encompassing yet fundamental theories ever developed in the history of physics, the Standard Model of particles. It is a theory which derives from the efforts to describe the fundamental particles behind all matter and forces that exist in nature. It was developed throughout the mid to late 20th century and includes twelve flavors of fermions - particles which follow Fermi-Dirac statistics - and five bosons - force carrier particles which follow Bose-Einstein statistics - Fig. 1.1.

The existence and specific properties of the Standard Model particles have implications which go beyond it and into the realm of cosmological and grand unification theories. Understanding the behavior of these particles has been the focus of particle physics experiments for decades.
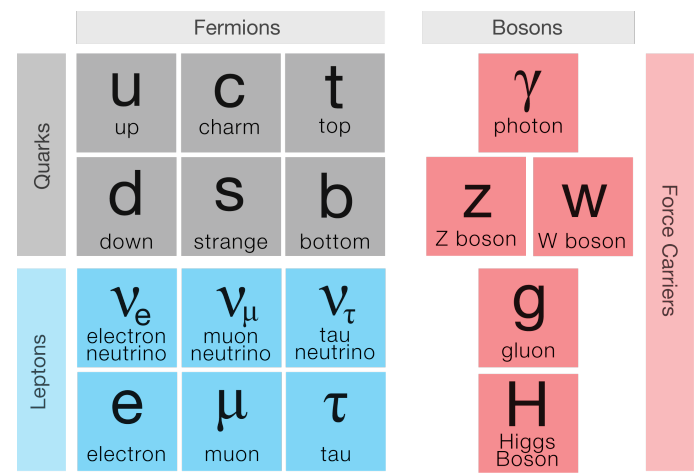

Figure 1.1: The collection of elementary particles predicted by the Standard Model. 
Neutrinos are particles predicted by the Standard Model. They are charge-less leptons with small masses which interact with other particles through the weak interaction. They are subject to neither the electromagnetic nor the strong forces. The existence of the neutrino was first proposed in the 1930's in order to develop an accurate description of beta decay without violating conservation of energy. However, the existence of multiple neutrino flavors was not predicted until after the discovery of a different flavor "electron-like" particle, the muon.

The electron neutrino $\nu_{e}$, the muon neutrino $\nu_{\mu}$ and the tau neutrino $\nu_{\tau}$ were discovered in 1956, 1962 and 2000, respectively, but have been found to have different properties than those predicted by the Standard Model. Neutrinos have small but non-zero masses, which contradicts their Standard Model description and gives rise to an unpredicted phenomenon for these particles called neutrino oscillations.

\section{$1.1 \quad$ Neutrino Oscillations}

Neutrinos are found in three different flavors in nature: $\nu_{e}, \nu_{\mu}$ and $\nu_{\tau}$. However, after a neutrino of a given flavor propagates in time it can be measured as a different flavor neutrino. This change of flavor of neutrinos in time is the phenomenon known as neutrino oscillations or neutrino mixing.

\subsubsection{Oscillations Theory}

The Pontecorvo-Maki-Nakagawa-Sakata (PMNS) matrix $U$ - also known as the neutrino mixing matrix - is a crucial expression to the understanding of the mixing of neutrinos. ${ }^{1}$

1 The expanded form of the PMNS matrix is multiplied by a diagonal matrix with elements corresponding to Majorana behavior of neutrinos which is a unit matrix for Dirac neutrinos. This is the assumption hereby imposed for this brief overview of the oscillation phenomenon. 


$$
\begin{aligned}
U & =\left(\begin{array}{lll}
U_{e 1} & U_{e 2} & U_{e 3} \\
U_{\mu 1} & U_{\mu 2} & U_{\mu 3} \\
U_{\tau 1} & U_{\tau 2} & U_{\tau 3}
\end{array}\right) \\
& =\left(\begin{array}{ccc}
-\operatorname{cin} \theta_{12} \cos \theta_{23}-\cos \theta_{12} \sin \theta_{13} \sin \theta_{23} e^{i \delta} & \cos \theta_{12} \cos \theta_{23}-\sin \theta_{12} \sin \theta_{13} \sin \theta_{23} e^{i \delta} & \sin \theta_{12} e^{-i \delta} \\
\sin \theta_{12} \sin \theta_{23}-\cos \theta_{12} \sin \theta_{13} \cos \theta_{23} e^{i \delta} & -\cos \theta_{12} \sin \theta_{23}-\sin \theta_{12} \sin \theta_{13} \cos \theta_{23} e^{i \delta} & \cos \theta_{13} \cos \theta_{23}
\end{array}\right)
\end{aligned}
$$

It relates their flavor eigensates (on the left) with their mass eigenstates (on the right)[9].

$$
\left|\begin{array}{c}
\nu_{e} \\
\nu_{\mu} \\
\nu_{\tau}
\end{array}\right\rangle=\left(\begin{array}{ccc}
U_{e 1}^{*} & U_{e 2}^{*} & U_{e 3}^{*} \\
U_{\mu 1}^{*} & U_{\mu 2}^{*} & U_{\mu 3}^{*} \\
U_{\tau 1}^{*} & U_{\tau 2}^{*} & U_{\tau 3}^{*}
\end{array}\right)\left|\begin{array}{l}
\nu_{1} \\
\nu_{2} \\
\nu_{3}
\end{array}\right\rangle
$$

The PMNS matrix allows for the flavor eigenstates to be written as superpositions of the mass eigenstates.

$\left|\nu_{\alpha}\right\rangle=\sum_{i} U_{\alpha i}^{*}\left|\nu_{i}\right\rangle$

such that the probability of oscillations can also be written in terms of the mass eigenstates.

$P\left(\nu_{\alpha} \rightarrow \nu_{\beta}\right)=\left|\left\langle\nu_{\beta} \mid \nu_{\alpha}(t)\right\rangle\right|^{2}=\sum_{j} \sum_{i}\left\langle\nu_{j}\left|U_{\beta j} U_{\alpha i}^{*}\right| \nu_{i}(t)\right\rangle$

is the probability of a $\nu_{\alpha}$ to be measured as a $\nu_{\beta}$ after some time t, during which it will have traveled a distance $\mathrm{L}$ in a vacuum.

This expression can be simplified and put in terms of the masses $m_{i}$ and energies $E_{i}$ of the mass eigenstates $\nu_{i}$ by describing their propagation with the plane-wave solution $\left|\nu_{i}(t)\right\rangle=e^{-i\left(E_{i} t-p_{i} x\right)}\left|\nu_{i}(0)\right\rangle$ for $\omega_{i}=E_{i} / \hbar$ and $k_{i}=p_{i} / \hbar$ in natural units. By introducing relativistic assumptions for the neutrino velocities, the time dependence can be re-stated in terms of $\mathrm{L}$ and the propagated eigenstate can be re-written as: 
$\left|\nu_{i}(t)\right\rangle=e^{-i m_{i}^{2} L / 2 E}\left|\nu_{i}(0)\right\rangle$

Such that the expression for the probability becomes:

$P\left(\nu_{\alpha} \rightarrow \nu_{\beta}\right)=\left|\sum_{i} U_{\beta i} U_{\alpha i}^{*} e^{-i m_{i}^{2} L / 2 E}\right|^{2}$

which is in terms of $m_{i}$ and the $\theta_{i j}$ and $\delta$ parameters from the PMNS matrix, as well as the $L / E$ factor, which is useful for experimental design for certain parameter assumptions (Fig. 1.4).

The following are two examples of the oscillation probabilities obtained from expanding Eqn. 1.6 with appropriate assumptions for the known allowed values of the $\theta_{i j}$ parameters (to leading-order).

$$
\begin{aligned}
P\left(\nu_{\mu} \rightarrow \nu_{e}\right) & \approx \sin ^{2}\left(2 \theta_{13}\right) \sin ^{2} \theta_{23} \sin ^{2} \Delta \\
& -\alpha \sin \left(2 \theta_{13}\right) \sin \delta \sin \left(2 \theta_{12}\right) \sin \left(2 \theta_{23}\right) \Delta \sin \Delta \sin \Delta \\
& +\alpha \sin \left(2 \theta_{13}\right) \cos \delta \sin \left(2 \theta_{12}\right) \sin \left(2 \theta_{23}\right) \Delta \sin \Delta \cos \Delta
\end{aligned}
$$

where $\Delta=\left(\Delta m^{2}{ }_{31} \frac{L}{4 E}\right) \quad \alpha=\frac{\Delta m^{2} 21}{\Delta m^{2} 31}$

$P\left(\nu_{\mu} \rightarrow \nu_{\mu}\right) \approx 1-\sin ^{2}\left(2 \theta_{23}\right) \sin ^{2}\left(1.27 \Delta m^{2}{ }_{32} L / E\right)$

These expressions are given in terms of the oscillation parameters $\theta_{i j}$ and $\delta$ introduced by the neutrino mixing matrix, as well as the difference between the squared masses of the mass eigenstates pertinent to this oscillation $\Delta m_{i j}^{2}$. While the PMNS matrix describes the phenomenon of neutrino oscillations, it does so in terms of the oscillation parameters. Experimental measurements of these parameters are necessary to truly understand neutrino oscillations and, thus, to constrain the theoretical implications of the phenomenon. 


\subsubsection{Experimental Measurements}

Neutrino experiments, such as $\mathrm{NO} \nu \mathrm{A}$, approach the challenge of measuring the mixing parameters by observation of the oscillations of individual neutrino flavors. The determination of individual oscillation probabilities (like those in Eqn. 1.7 and 1.8) for known values of $\mathrm{L} / \mathrm{E}$ conclusively constrains the possible allowed values of $\theta_{i j}$ and $\Delta m_{i j}^{2}$.

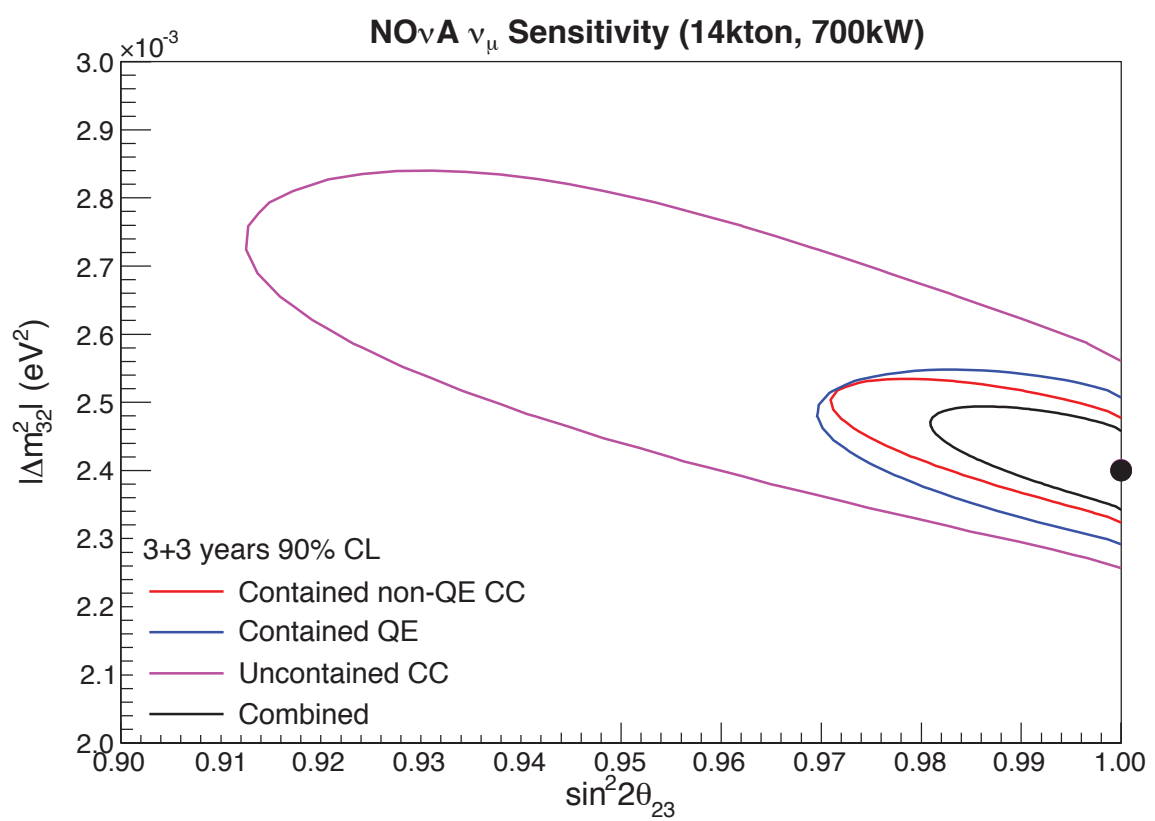

Figure 1.2: These contours show the sensitivity with which $\mathrm{NO} \nu \mathrm{A}$ is expected to constrain the parameters $\sin ^{2} 2 \theta_{23}$ and $\Delta m_{23}^{2}$ at $90 \%$ confidence level in the case that $\sin ^{2} 2 \theta_{23}=1$. These contours were produced by the $\mathrm{NO} \nu \mathrm{A}$ analysis groups using $\mathrm{MC}$ events equivalent to 3 years of neutrino and 3 years of antineutrino signal from the NuMI beam. The uncontained sample includes events with both escaping muons and uncontained hadronic showers. [1]

One approach at experimental study of neutrino oscillations is made through the use of man-made neutrino beams. Beam-line experiments consist of a controlled source of neutrinos which is propagated in a known direction and studied at two separate locations. The comparison of the specific-flavored neutrino energy distribution at the two points, separated by a distance $\mathrm{L}$, can be translated into a measurement of the oscillation probability. 
The task of measuring the parameters of the mixing matrix experimentally is essentially reduced to that of determining individual neutrino flavor energy distributions. Precision measurements of these distributions are, therefore, the main challenge in experiments like $\mathrm{NO} \nu \mathrm{A}$ because they translate into measurements of the oscillation parameters.

Given the small mass of neutrinos and their lack of electromagnetic and color charges, they interact very weakly with other particles. This makes it difficult to observe their behavior directly. Thus, the common methodology for studying neutrinos experimentally is to detect them indirectly by studying the product of their interactions with matter. The interaction of a neutrino with a nucleon produces more massive, sometimes charged particles which can be studied to reconstruct its energy.

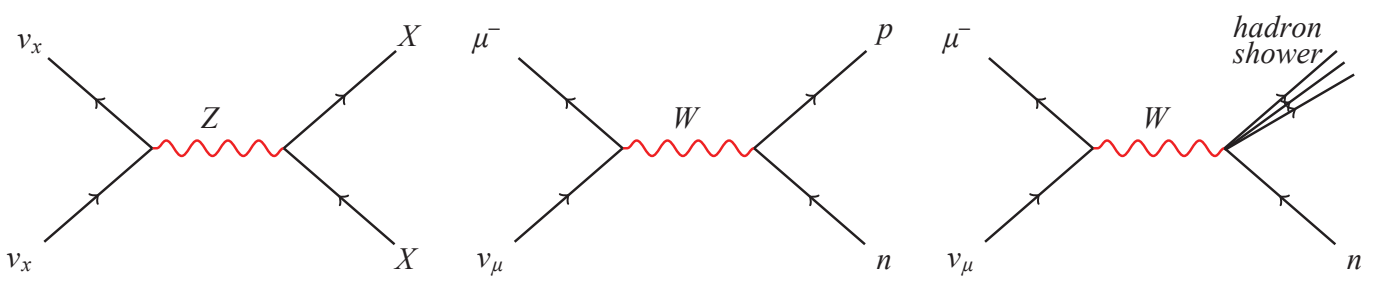

Figure 1.3: Left: Neutral Current $(\mathrm{NC}) \nu$ interaction. A $\nu$ interacts with a nucleon $\mathrm{X}$ and, through exchange of a neutral $\mathrm{Z}$ boson, a $\nu$ and a nucleon are produced. Center: Charge Current Quasi Elastic (CCQE) $\nu_{\mu}$ interaction. A $\nu_{\mu}$ interacts with a neutron and, through exchange of a charged $\mathrm{W}$ boson, a muon and a proton are produced. Right: $\nu_{\mu}$ Deep Inelastic Scattering (DIS). A $\nu_{\mu}$ interacts with a neutron and, through exchange of a $\mathrm{W}$ or $\mathrm{Z}$ boson a muon and a number of hadrons (hadronic shower) are created.

Figure 1.3 describes the main interactions neutrinos will undergo when traveling through matter. Due to energy and momentum conservation in these interactions, the energy of a neutrino can be reconstructed from the measured energy of its daughter particles. 
The studies discussed in this thesis are aimed towards energy measurements of one type of such daughter particles: muons. Muon energy reconstruction is the fundamental component in the reconstruction of $\nu_{\mu}$ energies and, thus, a key component in the oscillation analyses at $\mathrm{NO} \nu \mathrm{A}$.

\subsection{The $\mathrm{NO} \nu \mathrm{A}$ Experiment}

\subsubsection{Overview}

The NuMI Off-Axis $\nu_{e}$ Appearance Experiment $(\mathrm{NO} \nu \mathrm{A})$ is a long baseline neutrino experiment whose main physics goal is to search for $\nu_{\mu}$ to $\nu_{e}$ oscillations. NO $\nu \mathrm{A}$ studies neutrinos produced at the NuMI (Neutrino at the Main Injector) beam at Fermilab, a $700 \mathrm{~kW}$ neutrino beam directed towards the MINOS detector in Soudan, Minnesota. The experiment is comprised of two detectors in which the neutrino interactions are studied. One near detector underground (NDUG), close to the neutrino source and one far detector (FD) $810 \mathrm{~km}$ away and 14 mrad off-axis from the neutrino source [2].

The designed combination of properties from the neutrino beam energy spectrum and the specific location of the far detector (Fig. 1.4) allow $\mathrm{NO} \nu \mathrm{A}$ to probe the neutrino signal in conditions at which the oscillation probability for $\nu_{\mu}$ to $\nu_{e}$ is expected to be maximal. 


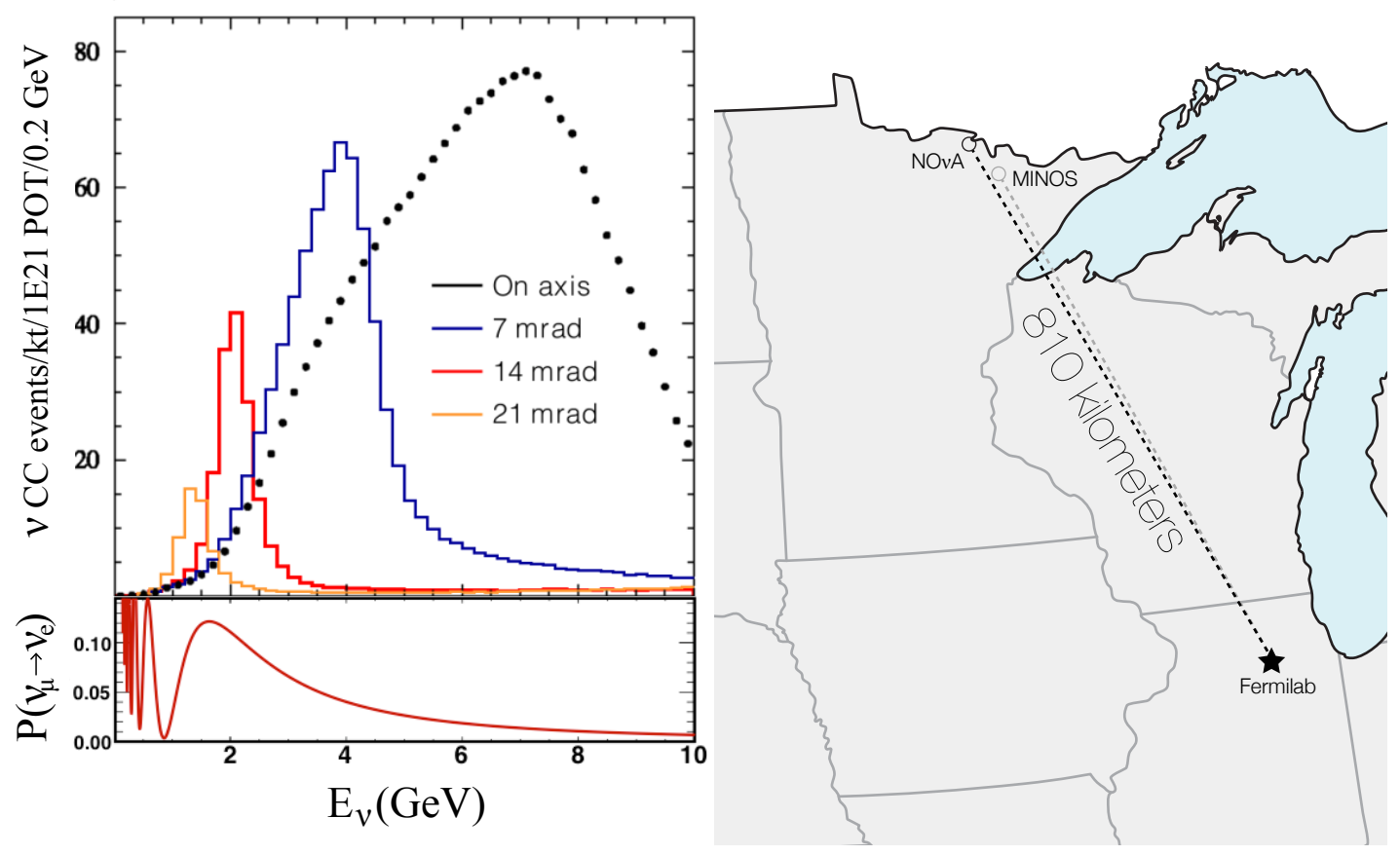

Figure 1.4: Distance and Angle Design Constraints for the NOvA Detectors. Left Above: The energy distribution for the NuMI beam neutrinos at different angles from the beam direction. The red line shows the energy distribution for the NO $\nu \mathrm{A}$ FD at $14 \mathrm{mrad}$. [2] Left Below: The oscillation probability $\mathrm{P}\left(\nu_{\mu} \rightarrow \nu_{e}\right)$ as a function of neutrino energy for $\mathrm{L}=810 \mathrm{~km}$, the distance from the NuMI beam source to the FD. Right: NO $\nu$ A detector locations, the NDUG at Fermilab and the FD $810 \mathrm{~km}$ from the source and 14 mrad from the beam direction.

\subsubsection{The NO $\nu \mathrm{A}$ Detectors}

The NO $\nu \mathrm{A}$ detectors are liquid scintillator based apparatus which facilitate the study of the particles produced in neutrino interactions which occur within them. They mainly consist of stacked polyvinyl chloride (PVG) tubular extrusion modules filled with liquid scintillator and outfitted with wave-shifting fibers. Figure 1.5 depicts a single tubular element of the $\mathrm{NO} \nu \mathrm{A}$ detectors. 


\section{Overview of the detection process:}

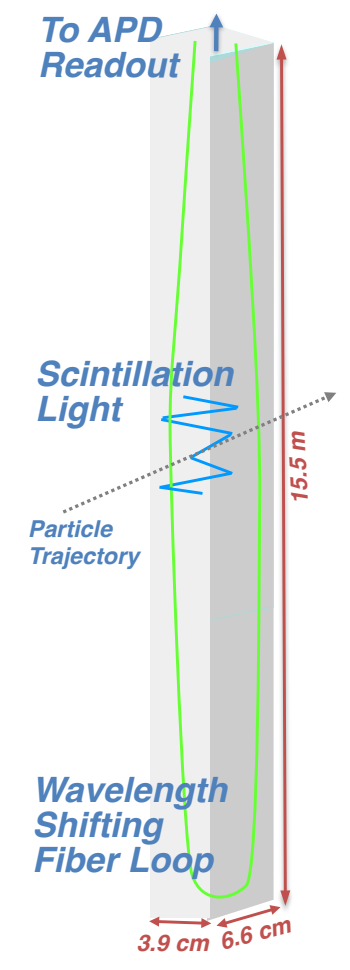

Figure 1.5: $\mathrm{NO} \nu \mathrm{A}$ detector element
1. A charged particle created from a neutrino interaction ionizes the scintillator material while traveling through it.

2. The light produced by the ionization is captured and reemitted by the wave-shifting component of the liquid at an appropriate wavelength for detection.

3. The reemitted light travels and is reflected by the PVC walls until absorbed by the fiber.

4. The signal travels through the fiber and into the electronics, which convert and store the signal appropriately.

5. Once stored, attenuation and calibration corrections are made on the signal, which can be converted into energy units.

6. Ultimately, the signal is stored in units equivalent to the amount of energy deposited by the charged particle in the cell.
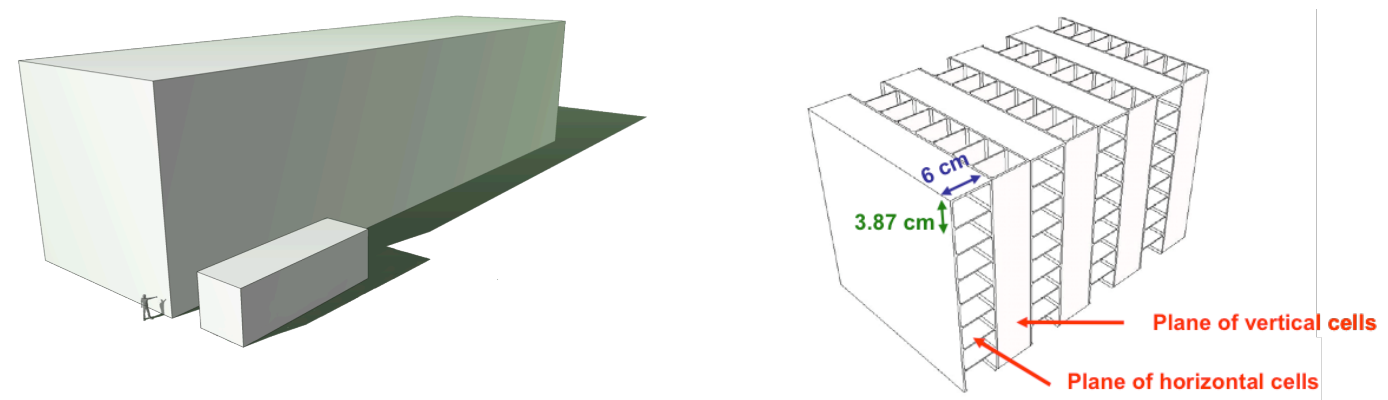

Figure 1.6: Detector Structure. Alternating stacked PVG modules make up the rigid structure of the detectors $(\sim 15.5 \times 15.5$ meter planes for the FD and $\sim 4.2 \times 4.2$ meters for the NDUG). 


\subsection{3 $\nu_{\mu}$ Oscillation Measurements}

The NO $\nu \mathrm{A}$ experiment is capable of studying two oscillation probabilities of the $\nu_{\mu}$. The $\nu_{e}$ appearance or $P\left(\nu_{\mu} \rightarrow \nu_{e}\right)$ and the $\nu_{\mu}$ disappearance or $P\left(\nu_{\mu} \rightarrow \nu_{\mu}\right)$ probabilities. As mentioned in Sec. 1.1.2 the energy distributions of the $\nu_{\mu}$ beam at the NDUG and FD are essential to the oscillation analyses that NO $\nu$ A will undertake. The oscillation probabilities are obtained through the comparison of the energy distributions for $\nu_{\mu}$ at the NDUG and those for $\nu_{\mu}$ and $\nu_{e}$ at the FD for $P\left(\nu_{\mu} \rightarrow \nu_{\mu}\right)$ and $P\left(\nu_{\mu} \rightarrow \nu_{e}\right)$, respectively. Figure 1.7 shows this comparison for simulations of the NuMI beam at $\mathrm{NO} \nu \mathrm{A}$ with assumed oscillation parameters.

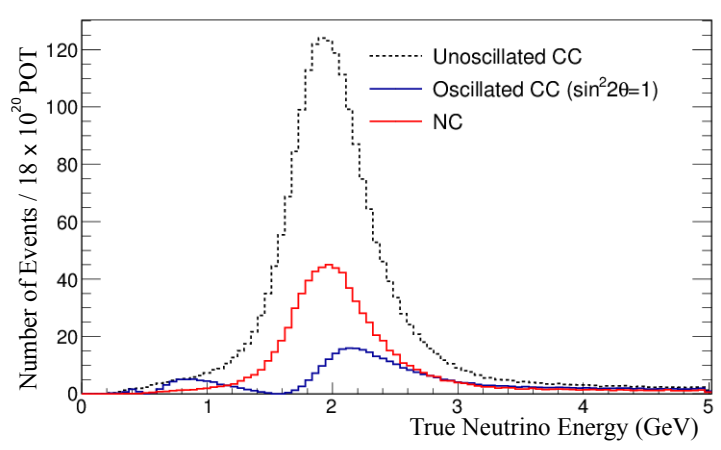

Figure 1.7: Expected unoscillated and oscillated (disappearing) $\nu_{\mu}$ signal for $\mathrm{NO} \nu \mathrm{A}$ with 3 years of neutrino data and 3 years of anti-neutrino data.

The measurements of these energy distributions are made through direct detection of the daughter particles from the interactions and the use of conservation of energy. For example, in the case of muon neutrino CCQE interactions (Fig. 1.3) the energy of the incident neutrino is given by:

$E_{\nu_{\mu}}=E_{\mu}+E_{p}-E_{n}$

The neutrons in the interaction are nucleons within the detector material. Thus, they are moving at negligible velocities and can be considered to have known energy $E_{n}=m_{n} c^{2}$. The energy of the protons can be estimated with some certainty by summing over the energy deposited in the detector cells. In the case of the muons, their behavior and energy reconstruction methodology will be discussed in chapter 2 . 


\section{Chapter 2}

\section{Muons in the NO $\nu$ A Detectors}

\subsection{Muon Behavior in the Detectors}

Muons are leptons described by the Standard Model which were discovered in 1936 during cosmic radiation studies. They are electron-like particles with rest mass of approximately $105 \mathrm{MeV} / \mathrm{c}^{2}$ and the same values for charge and spin as electrons. As discussed in Sec. 1.1.2, muons are found in the $\mathrm{NO} \nu \mathrm{A}$ detectors as products of neutrino interactions, although the most abundant source of muons in the detectors are those produced by cosmic ray interactions in the atmosphere. Understanding the behavior of muons in the detectors is not only a crucial component of the oscillation measurements but equally important for any studies regarding cosmic ray muons as well.

"Moderately relativistic charged particles other than electrons lose energy in matter primarily by ionization and atomic excitation" [3]. As a muon travels through the detector it will deposit all of its energy mostly by these means. The curve shown in Fig. 2.1 relates the amount of energy deposited by a particle per unit length traveled in matter $(d E / d x)$ with its velocity. 
Given that the range of momenta (and thus, velocities) of the muons in our expected signal is known, this relation plot can be used to predict their expected $d E / d x$ range as well. The mean value for the energy of beam neutrinos for $\mathrm{NO} \nu \mathrm{A}$ is $2 \mathrm{GeV}$ (Fig. 1.4). Assuming a range in muon momentum within an order of magnitude around $1 \mathrm{GeV} / \mathrm{c}$ one can clearly notice that muons from neutrino interactions in $\mathrm{NO} \nu \mathrm{A}$ are well within the minimum ionization region in Fig. 2.1. ${ }^{1}$ Because the muons of interest will be min-

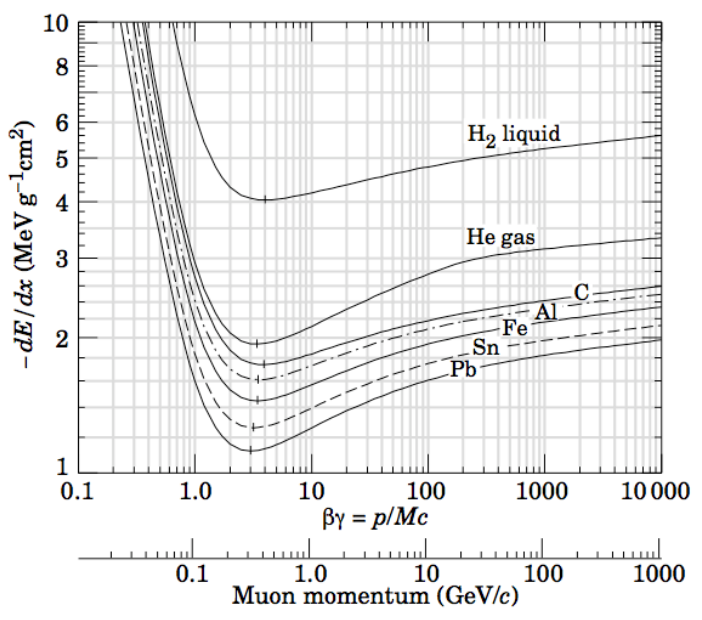

Figure 2.1: Muon $\mathrm{dE} / \mathrm{dx}$, described by the BetheBloch curves. [3] The curve for $\mathrm{C}$ is a good approximation for the material in the $\mathrm{NO} \nu \mathrm{A}$ detectors. imum ionizing, they will travel long distances within the detector before depositing their energy entirely. These traveling distances will be related to their initial energy as well.

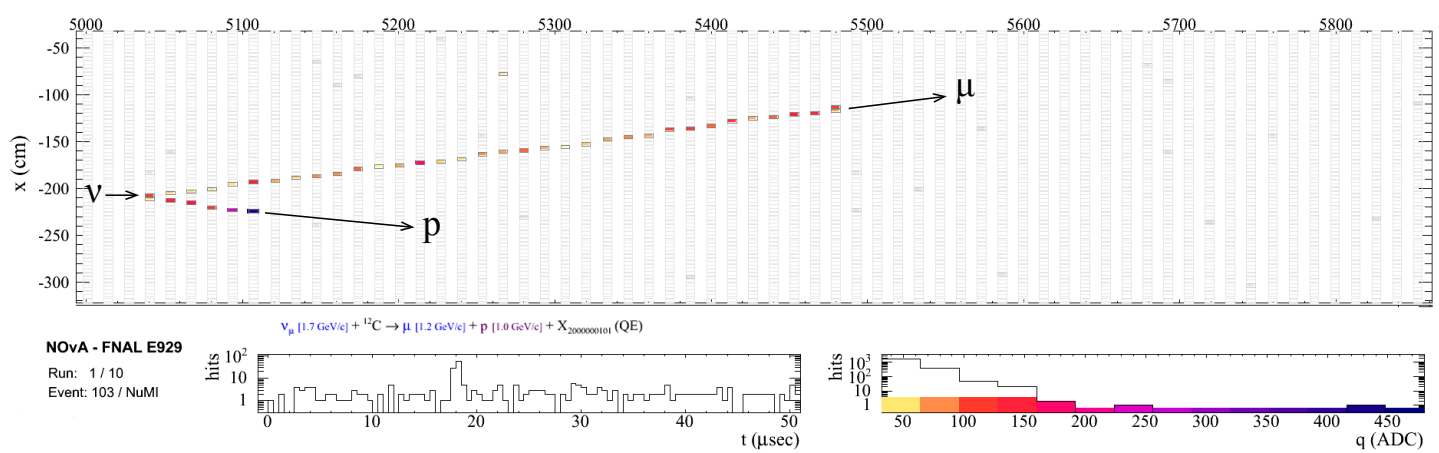

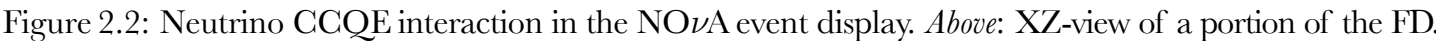

The hits for the proton and the muon produced in a $\nu_{\mu}$ interaction are colored to signal energy deposition in the cell. Below: The event details histograms showing the activity in this section of the detector in the displayed time window and the color coded ADC counts (proportional to the deposited energy).

1 It might appear to the reader as though the terms momentum and energy are used interchangeably. It is important to note the reason for this apparent misuse. Given that the particles being dealt with are relativistic, their energy can be calculated with the equation $E^{2}=\left(m c^{2}\right)^{2}+p^{2} c^{2}$. While finding the momentum and energy of a particle are not equivalent, given that the rest mass of the muon is known, behavior correlated to its energy is, thus, correlated to its momentum; and measurement of one of these quantities can be easily translated into the other. 


\subsection{Muon Energy Reconstruction}

The quantity of interest for the oscillation analysis is the muon's energy, as seen in Eq. 1.9. Thus, the focus of reconstructing the characteristics of muons which travel within the detector is on determining their energy. ${ }^{2}$

\subsubsection{Reconstruction Methodology}

Intuitively, the simplest way to reconstruct a particle's energy consists of summing over the energy it deposits in each cell while traveling through the detector. However, there is a major disadvantage to this reconstruction method when applied to muons. As minimum ionizing particles, muons travel larger distances in the detector and deposit a non-negligible percentage of their energy on the module walls as they traverse them. Given that only the inside of the tubular structures is instrumented for detection, energy deposited in the PVC is undetectable. Consequently it cannot be assumed that the addition over the observable deposited energy would account for the muon's energy entirely. As seen in Fig. 2.3, this method would result, in general, in an underestimation of the particle's energy.

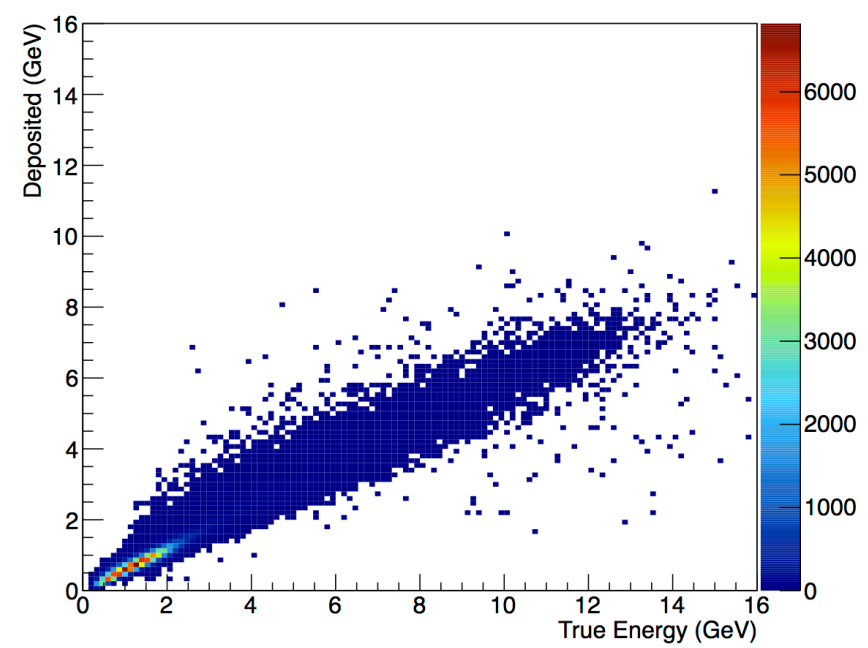

Figure 2.3: Correlation between summed deposited energy and true muon energy.

2 The term "particle's energy" is generally used in this text to refer to initial energy. That is, the energy of the particle as it is created in the interaction or, in the case of neutrinos, as it interacts in the detector. 
A far more reliable method used in $\mathrm{NO} \nu \mathrm{A}$ to reconstruct muon energies is the "track length method". As muons' paths through the detector are only long enough for all their energy to be deposited in it, these lengths are surely correlated with their energy. Figure 2.4 shows this correlation for simulated muon events in the FD. This method of reconstruction consists of quantifying the correlation by fitting a mathematical relation to its profile and utilizing that relation. In the case of this correlation a linear function suffices to describe its profile. The reconstructed energy itself is then calculated by evaluating the function for the given value of track length for each muon. ${ }^{3}$
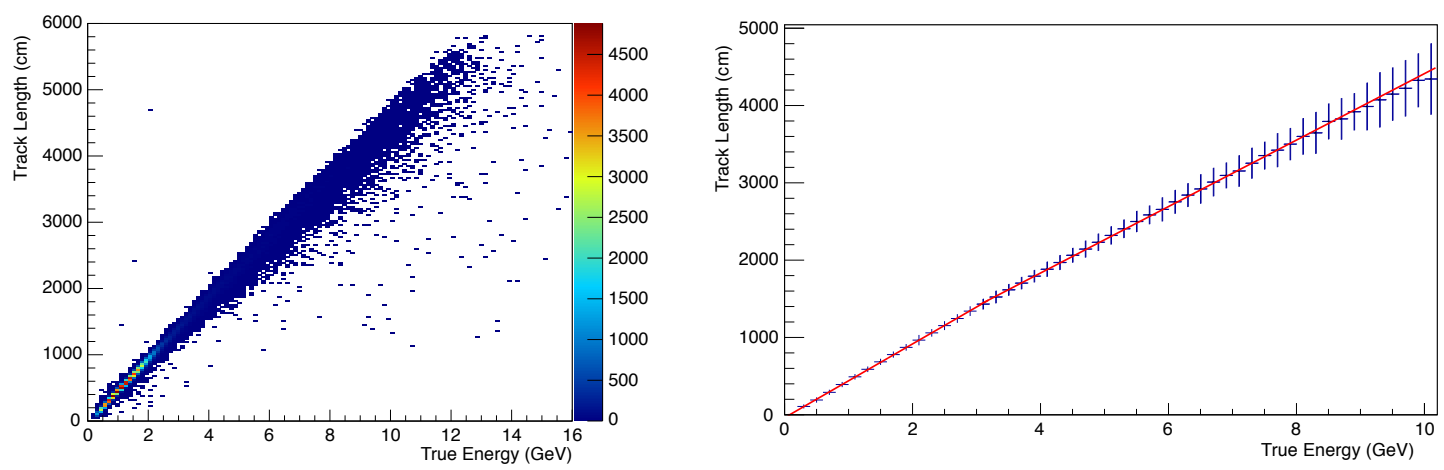

Figure 2.4: Left: Correlation between muon track length and true energy. Right: Correlation profile and linear fit which describes the correlation and is used for reconstruction.

\subsubsection{Reconstruction Method Evaluation}

The correlation shown in Fig. 2.4 indicates that the track length method can be a useful gauge of muon energy. Such "usefulness" can be assessed in various ways to allow the comparison of different approaches at reconstruction. A qualitative evaluation of the track length method is shown in Fig. 2.5. Ideal energy reconstruction would yield a perfect linear correlation between the reconstructed and true energy values on this distribution.

3 The actual approach that is currently employed in the $\mathrm{NO} \nu \mathrm{A}$ software involves not one but various linear fits to predefined sections of the shown correlation, respectively. The details of this approach, however, are continuously being improved and unnecessary for the discussion presented in this thesis. 

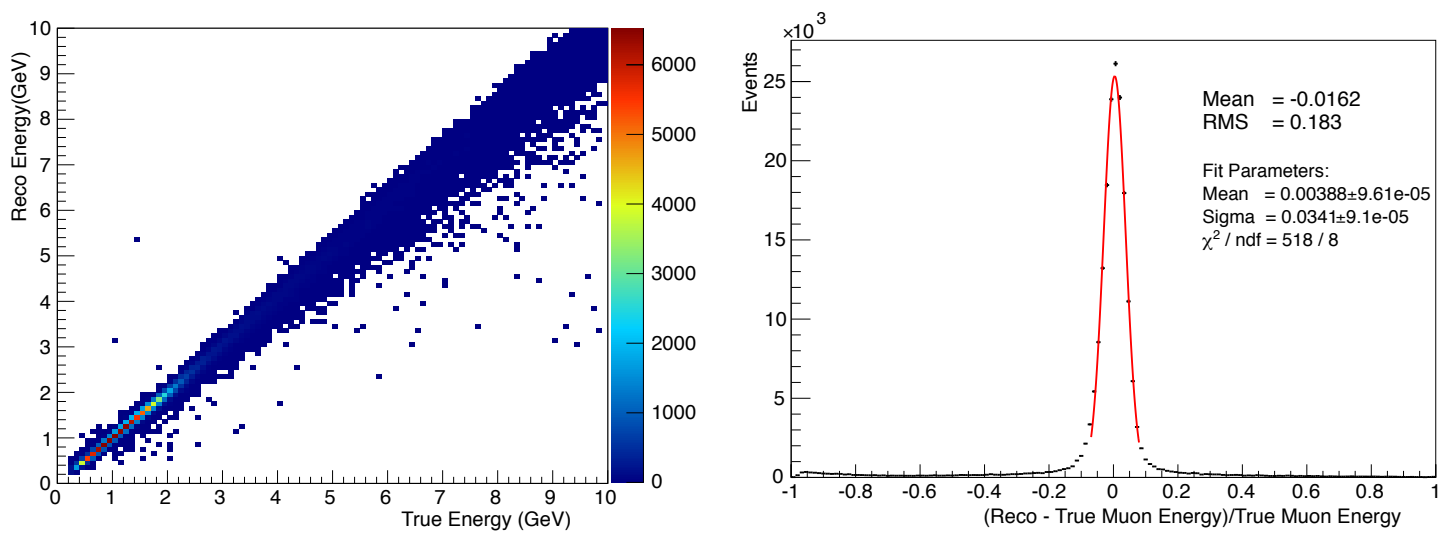

Figure 2.5: Evaluation of the Track Lenth Reconstruction Method. Left: Qualitative evaluation. A visual comparison can be made to determine some qualities of the method. Right: Quantitative evaluation. The qualitative evaluation plot's profile and gaussian fit (red), whose standard deviation (sigma) gives a measure of the method's resolution.

A quantitative approach at evaluating the method can be seen in Fig. 2.5. A distribution of the percent difference between the reconstructed and true muon energy is shown. Such distribution can be fitted to a gaussian function whose width, given by it's standard deviation, gives a measure of the method's energy resolution. Ideal energy reconstruction would yield a scaled delta function with height determined by the number of entries in the distribution.

\subsection{Escaping muons}

Given its simplicity, the track length method constitutes a useful example to explain the concepts of energy reconstruction and resolution. While this method is not that with which this thesis is concerned, it is in its limitations that the motivation for this work arises.

To achieve the well understood performance of the track length method it requires only that the length of the muon's trajectory is known. However, this information is not 
available for a fraction of the muons from neutrino interactions in the detectors. Such are those muons which are produced within the detector but exit it before having deposited all their energy. These "escaping muons" will constitute approximately $28 \%$ of all muons from neutrino interactions in the FD with energies between 1 and $4 \mathrm{GeV}$.

The importance of escaping muons relies on the amount of statistics that are typical to neutrino experiments. The extremely small interaction cross section of neutrinos with matter, around $10^{-38} \mathrm{~cm}^{2}$ per nucleon for single $\mathrm{GeV}$ energy neutrinos in carbon[3], results in low statistics per unit time in experiments like $\mathrm{NO} \nu \mathrm{A}$. Given that the expected rate of neutrino interactions in the FD is less than 10 per day, the use of as much of the available data as possible is of extreme importance to the oscillation analyses.

The objective of the work presented in this thesis is to explore one possible approach at utilizing data from escaping muons. By reconstructing their energies through a method which does not rely on complete track length information, the sample of escaping muons can be incorporated into the analyses, albeit with less resolution than the fully contained sample.

\subsection{Muon Multiple Scattering}

In addition to depositing energy, muons undergo multiple scattering as they travel through the detector. This scattering is caused by the coulomb force exerted by the protons in an atomic nucleus on a closely passing muon. Given that muons from neutrino interactions travel at relativistic speeds through the detector material, these forces are small compared to their momentum causing only tiny deviations from the particle's original trajectory.

The characteristics of individual scattering events along a muon's path are dependent on its distance to the nucleon as well as its momentum and the total charge of the nucleon itself. Thus, calculating these small scattering angles a priori would require precise 
knowledge of the atomic array within the detector as well as the direction of travel of the muon with atomic length precision. There is, however, a more sensible expression for the angles of multiple scattering that relates them to the muon's behavior as well as the known characteristics of the media through which it travels.

$\theta_{0} \approx \frac{13.6}{(p c \beta)} \times \sqrt{\frac{x}{X_{0}}} \times\left[1+0.038 \ln \left(\frac{x}{X_{0}}\right)\right]$

Equation 2.1[4] is not an expression for individual scattering angles but for the distribution of angles at which the muon will be deflected. While traveling a distance $x$ in a material with radiation length $X_{0}$, a muon with momentum $\mathrm{p}$ and velocity $\beta c$ will undergo multiple scattering at angles described by a gaussian distribution about zero and with standard deviation $\theta_{0}$. For the specific case of muons inside the $\mathrm{NO} \nu \mathrm{A}$ detectors this distribution can be calculated with the known radiation length of $44 \mathrm{~cm}$ for the detector material $^{4}$ and an approximation for relativistic muons of $\beta \simeq 1$.

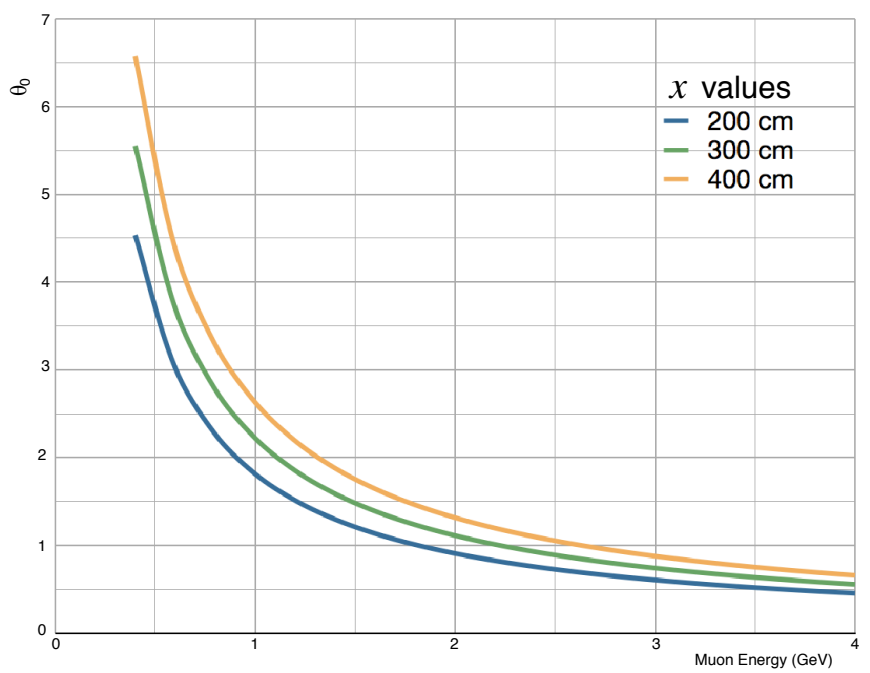

Figure 2.6: Expected $\theta_{0}$ vs energy distributions for relativistic muons in the $\mathrm{NO} \nu \mathrm{A}$ detector. From Eq. 2.1 with $X_{0}=44 \mathrm{~cm}$ and $\beta=1$.

\footnotetext{
${ }^{4}$ While the definition of radiation length is expressed in units of $\mathrm{g} \mathrm{cm}^{-2}$ it is common to see it expressed in $\mathrm{cm}$, the radiation length divided by the density of the material.
} 


\subsubsection{Multiple Scattering and Energy Reconstruction}

The correlation between a muon's energy and its distribution of scattering angles implies some potential for energy reconstruction. Section 2.2 explains how a measurement of a quantity with some correlation to the energy can be used for its reconstruction. In the same way, the measurement of multiple scattering could yield information about the energy of muons in the detector

The concept of energy reconstruction via multiple scattering has been employed before in other neutrino experiments $[4,5]$. However, the particular characteristics of the $\mathrm{NO} \nu \mathrm{A}$ detectors - their geometry, composition and resolution capabilities - impose unique constraints on the studies of multiple scattering within them. Thus, the task of measuring muon scattering in $\mathrm{NO} \nu \mathrm{A}$ is notably different from what has been done in other experiments, as is that of determining its usefulness to make energy estimations.

It is clear that Fig. 2.6 does not show as pronounced a correlation as those in the methods described in Sec. 2.2. Thus, a method which relies on it for energy reconstruction might not yield as good a resolution as those previously discussed. However, the potential of multiple scattering based reconstruction lies not in its resolving power, but in its possible usefulness when applied to escaping muon events. Given that $\theta_{0}$ in Eq. 2.1 is given in terms of the distance traveled by the particle, it is implicit that it can be employed when examining portions of, and not necessarily entire particle trajectories. As opposed to the track length and energy deposition methods, a multiple scattering method would have the potential to make energy estimations for escaping, as well as contained, muons in the detector.

The main objectives of the project presented on this thesis are thus to study muon multiple scattering in the $\mathrm{NO} \nu \mathrm{A}$ detectors; to develop a technique to reconstruct muon energies using multiple scattering information and to evaluate this method's possible 
usefulness in the manner shown in Sec. 2.2.2. The following chapters contain a description of the methodology employed to accomplish these objectives, the results of which are presented in Sec. 4. 


\section{Chapter 3}

\section{Scattering Angle Reconstruction}

The essential feature required in order to study multiple scattering is the ability to measure the small deviations which occur in the muons' trajectories. The task of measuring these deviations within the $\mathrm{NO} \nu$ A detectors, however, is anything but straightforward.

Given the granularity of the detectors shown in Fig. 1.6 and the detection process outlined in Sec. 1.2.2, the elementary pieces of information that the detector collects are amounts of energy deposited in individual cells in the detector at a given time. This results in a resolving power for the particles position at any point of its trajectory of within the order of centimeters.

As a result, the measurement of individual scattering occurrences is infeasible, considering the small nature of the expected deviations (Fig. 2.6). This does not suggest, however, that obtaining any information of these scatterings is unattainable. While some small scatterings will undeniably be lost in the limitations of our spatial resolution, others will have a noticeable effect on the particle's path. The conditions that cause a single scattering event to impact the particle's trajectory measurably (or not) are simply geometrical and are shown schematically in Fig. 3.1. 


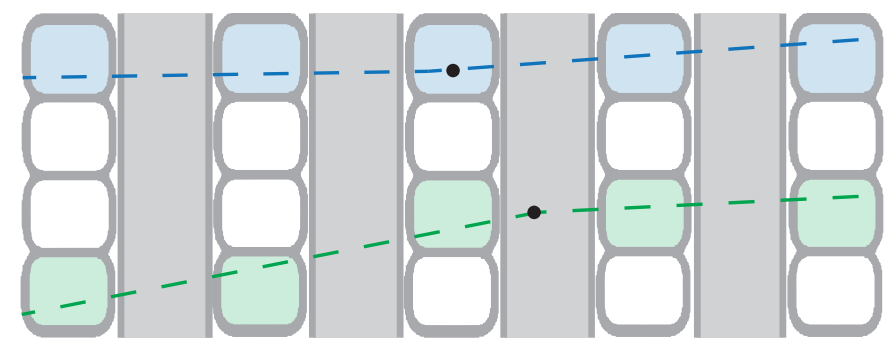

Figure 3.1: Diagram depicting the potential impact of small scatterings on a track. Particle trajectories given by the dotted lines and produce visible hits on colored cells. A single scattering event at small angles will not necessarily deviate the particle enough for any change in its trajectory to be noticeable within the detector's granularity (blue).

\subsection{Reconstructed Track Structure}

The task of extracting scattering information from known hits - single data of energy deposition in a cell - is done through the examination of the estimated path followed by the particle. This path, called a reconstructed track, is outlined by the NO $\nu \mathrm{A}$ software using position information from multiple hits which are associated with the same particle. ${ }^{1}$

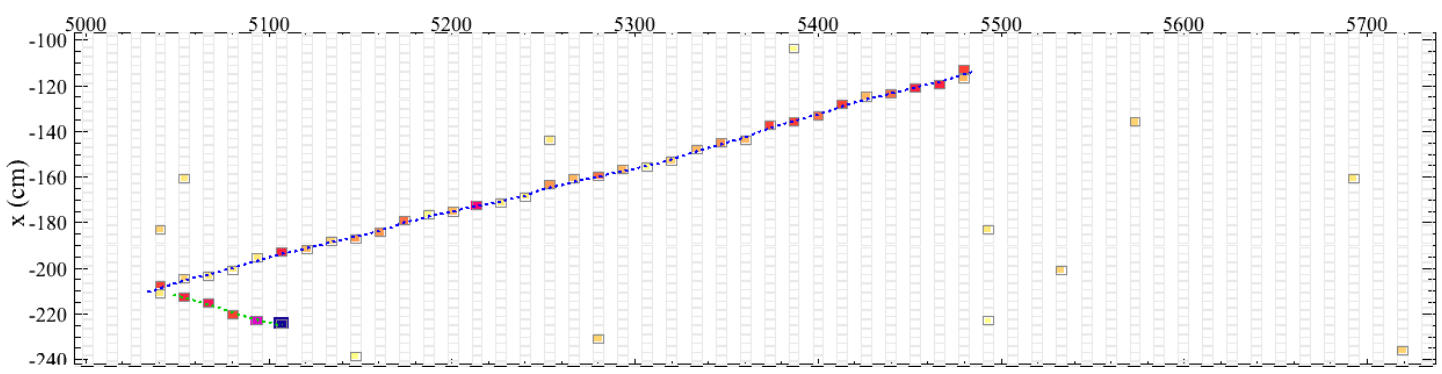

Figure 3.2: Reconstructed tracks in the event display. The dotted lines overlaid on the hits follow the estimated path of the particle as outlined by the reconstruction software which groups hits by spacial and time coincidence and outlines the tracks.

\footnotetext{
1 A given set of hits is associated with having been produced by the same particle provided that they coincide in a given time window and are related to one another spatially.
} 
Careful inspection of the reconstructed track for the muon hits in Fig. 3.2 (blue line) reveals some information about the tracker algorithm. ${ }^{2}$ The tracker builds a path which matches all the positions implied by individual cell hits, however, it does so by outlining a "smooth" path rather than discrete line segments. Ultimately, in the software environment used for data analysis in $\mathrm{NO} \nu \mathrm{A}$, reconstructed tracks are $\mathrm{C}++$ objects which contain a set of "trajectory points" which describe the path. The number of trajectory points in a track is equal to the number of hits used to outline it, such that each individual trajectory point represents the position in space at which a particle produced its associated hit.

\subsection{Extracting Scattering Angles from Tracks}

In order to measure the detectable deviations within a track, it is divided into fragments which will be referred to as "vectors". The angles of interest are given by the angles between every pair of consecutive vectors along a track.

Initially, vectors were defined as straight lines connecting two consecutive trajectory points as shown in Fig. 3.3. However, more careful examination of the tracker behavior revealed that the "smooth" shape of the reconstructed track introduces small curvatures to the particle's path. This feature of reconstructed tracks would result in the measuring of angles unrelated to scattering

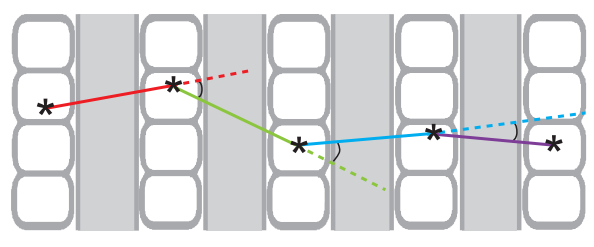

Figure 3.3: Angle measurements are done by defining vectors (color lines) as straight lines between trajectory points (stars) and calculating the angle between consecutive vectors. with this simple methodology.

2 Track reconstruction is a functionality developed within the NO $\nu \mathrm{A}$ collaboration and not through the work presented in this document. 


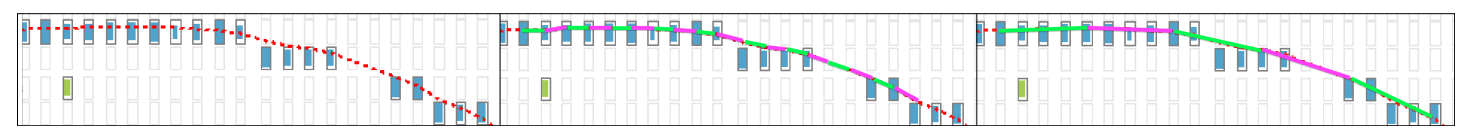

Figure 3.4: Left: A magnified track portion which depicts the slight curvature introduced by the tracker. Center: The first approach at angle measurement with plane-to-plane vectors. Right: Angle measurement with larger vectors avoids the measurement of the "fake-scattering" angles.

These "fake scattering" angles are small features of the track which plane-to-plane analysis will detect. However, if the number of planes spanned by the vectors - vector size - is increased, the detection of fake scatterings will be reduced and only true scatterings which impact the particle's trajectory noticeably will be detected (Fig. 3.4).

The increase in vector sizes from one to many planes undeniably constrains our ability to detect very small scatterings. However, it enhances the ability to detect those which are not artificially created and, thus, more likely to be correlated with the muon's energy. The vector size used for the rest of the studies and the energy estimations was that which maximized the apparent energy correlations which are discussed in Sec. 3.3.2. Ultimately, the optimal vector size was determined to be six planes.

It is important to note that the methodology for the measurement of angles is slightly different for the case of vector sizes larger than one plane. In addition to dividing the track into vectors spanning six planes each, this process was repeated for the same track with the first vector starting on the second, third, and so on up to the (vector-size -1) trajectory point. In this way, a scattering event occurring at any given plane will have a pair of vectors which measure it with respect to the expected track direction right before and right after the deviation. 


\subsection{Angle to Energy Correlations}

The following step towards making energy estimations was to examine the correlations found between the measured scattering and the energy of muons. This was accomplished by measuring multiple scattering on a sample of simulated muons in the detector.

Appendix A discusses the process of generating such simulation and the parameters chosen to define it. In the software environment, the sample of simulated muons is identical in structure to the data that the detector and reconstruction software would be expected to yield when detecting such muons. In addition, the simulations contain "MC Truth"-Monte Carlo simulation "truth" - information which includes parameters like true energy and position of the simulated particles and other data known from the simulation but independent of the detector and software performance.

The process described in section 3.2 is used to determine a collection of angles for each muon track in the simulation. The standard deviation of such collection of angles, denominated "angleSigma", is calculated for each muon track. The distribution of angleSigma with respect to true muon energy - the individual particle energy known by MC truth - will depict the correlation between our measure of scattering and a particle's energy, if any is found.

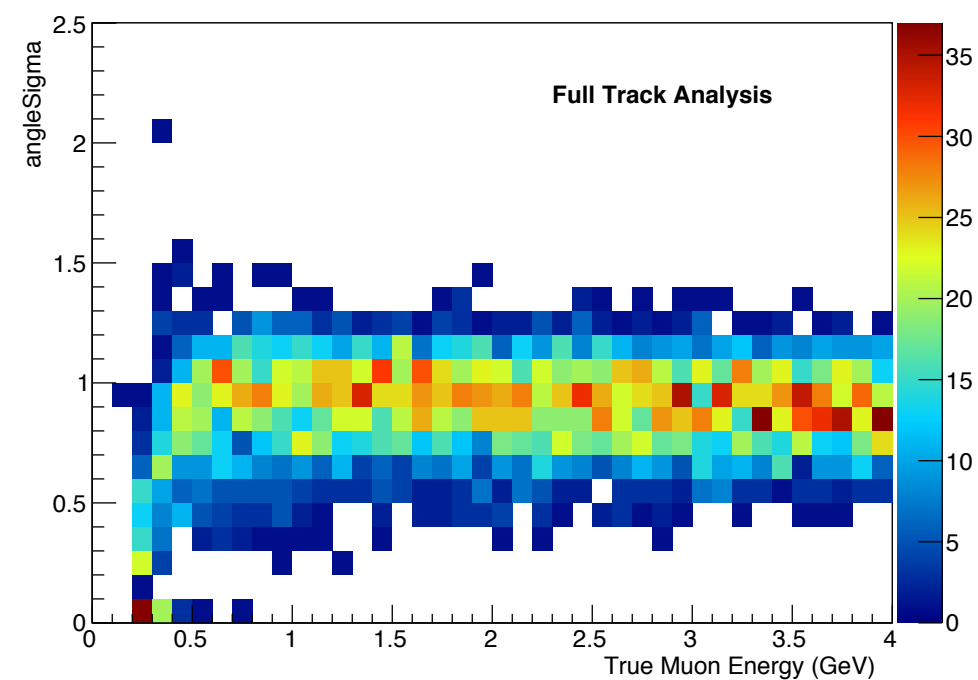

Figure 3.5: Distribution of angleSigma vs true muon energy. No correlation is found for angleSigma calculated with angles from the full length of the track. 
Further examination of the procedure employed to obtain this correlation revealed a clear discrepancy between the data used to produce Fig. 3.5 and the prediction obtained from Eq. 2.1, which translates into a correlation like that shown in Fig. 2.6. In Eq. 2.1, $\theta_{0}$ is given as a function of $x$, the distance traveled by the particle. Thus, the correlation it implies is valid for $\theta_{0}$ obtained from the scattering of particles through equal distances; while the data shown uses values of $\theta_{0}$ from angles obtained from the entire track.

As muons travel through the detector they deposit energy. Thus, examining the behavior of the last piece of a long muon track is equivalent to doing so for the shorter track of a lower energy muon. $\theta_{0}$ in Fig. 3.5 then reflects behavior from the entire trajectory of a muon and in doing so it smears information not only from the initial energy of the particle but from sections of the track which are characteristic to lower energy muons.

\subsubsection{Measurements using track segments}

In order to avoid the smearing of scattering information and to obtain distributions which compare equal distances traveled by muons, the same angle measuring procedure is now used for track segments of equal length. The distribution shown in Fig. 3.6 is obtained by using data from track segments 4 meters in length, from the beginning of the track. This distribution shows a clear change in the behavior of the angleSigma variable with respect to the muon energy, which agrees with the expected correlation. ${ }^{3}$

3 Given that the scattering angles are not measured individually, it is incorrect to assume that angleSigma and $\theta_{0}$ are equivalent. While angleSigma is a standard deviation of the measured angles, it also displays behavior related to the detector's spatial resolution and potential double counting of some angles by the definition of vectors. However, this variable still contains scattering-related behavior captured by the track reconstruction and a similar correlation with the energy is expected. 


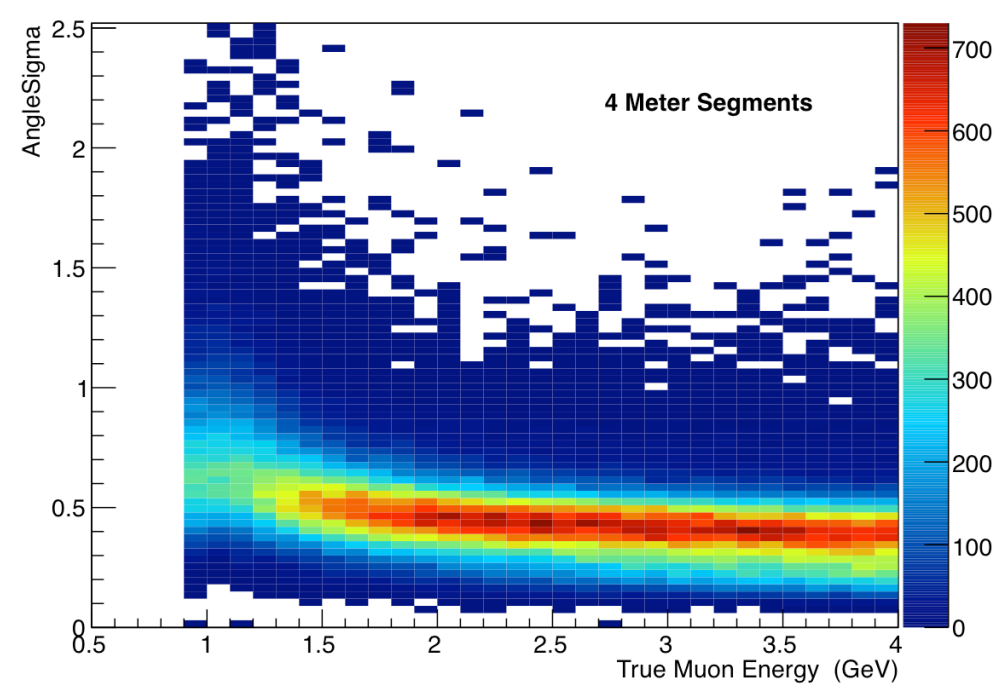

Figure 3.6: Correlation found between angleSigma from 4 meter segments and true muon energy

While the analysis of track segments diminishes the smearing given by full track analysis, it also introduces an additional parameter which must be fixed to make equivalent comparisons: the segment length. The conundrum encountered when choosing segment lengths relies on the fact that shorter segment analysis yields information which is more characteristic to the particle's initial energy, but it also contains less information as fewer vectors can be defined within a shorter segment.

The segment length to be used for further analysis and reconstruction was fixed by comparing the correlations obtained for various lengths. This parameter, as well as the vector size, were fixed to those which yielded more visible correlations between angleSigma and the energy. In the case of vector size its value was fixed to six planes.

Segment lengths of 2, 3 and 4 meters were analyzed and yielded the correlations shown in Fig. 3.7. While there are observable correlations in all cases, a segment length of 4 meters was chosen for the rest of the analysis given that it yields more visibly pronounced correlations around the $2 \mathrm{GeV}$ energy peak. It is important to consider the implications of such choice for the segment length, however. Any given segment length necessarily 
constrains the minimum total track length which can be used for energy reconstruction. Nevertheless, given that the ultimate objective of this method is to reconstruct escaping muon events, shorter tracks are less likely to be part of this target sample. As such, the discrimination of this method towards shorter tracks is not of immediate concern.
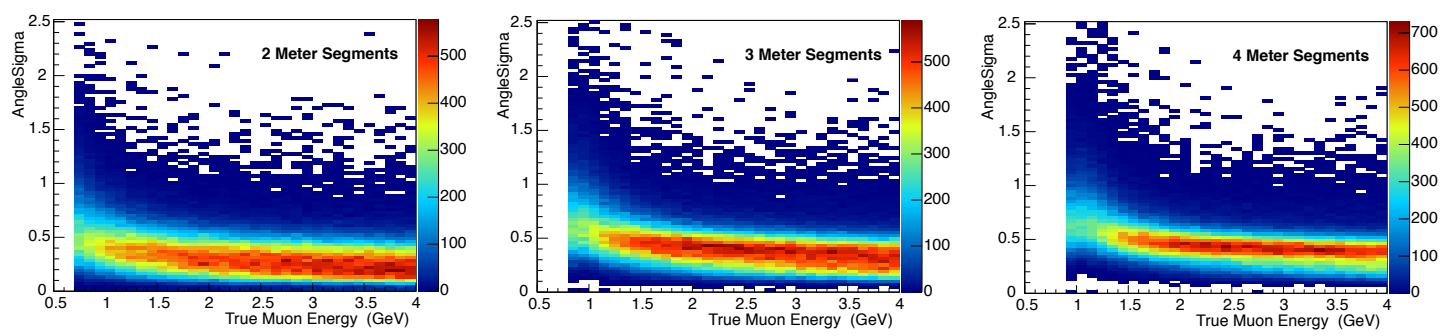

Figure 3.7: Correlation found between angleSigma and true muon energy for different segment lengths. These distributions were compared in order to fix the value of "segment length" to that which yields the most visible correlation.

\subsubsection{Gauging Multiple Scattering}

The description of multiple scattering given by Eq. 2.1 implies a correlation between the angles' standard deviation and the energy. However, angleSigma might not be the definitive gauge on muon multiple scattering behavior.

In order to utilize all available information which reflects the concept that higher energy muons are more likely to scatter at smaller angles, three additional variables are introduced as gauges of multiple scattering:

angleSum - the addition of all angles found along a track

angleMax - the largest of such angles

angleExt - the angle between the first and last vector along the track segment 
The correlations between these new variables and the energy, shown in Fig. 3.8 exhibit similarly shaped correlations, which is expected given that they are all measures of the same phenomenon - multiple scattering. However, the specific correlations are visibly different from one another. This implies that they indeed capture slightly different features of either the scattering behavior or its correlation with the energy. The following chapter discusses the usage of these correlations to reconstruct the energy of muons and the approaches used to exploit the differences between them.
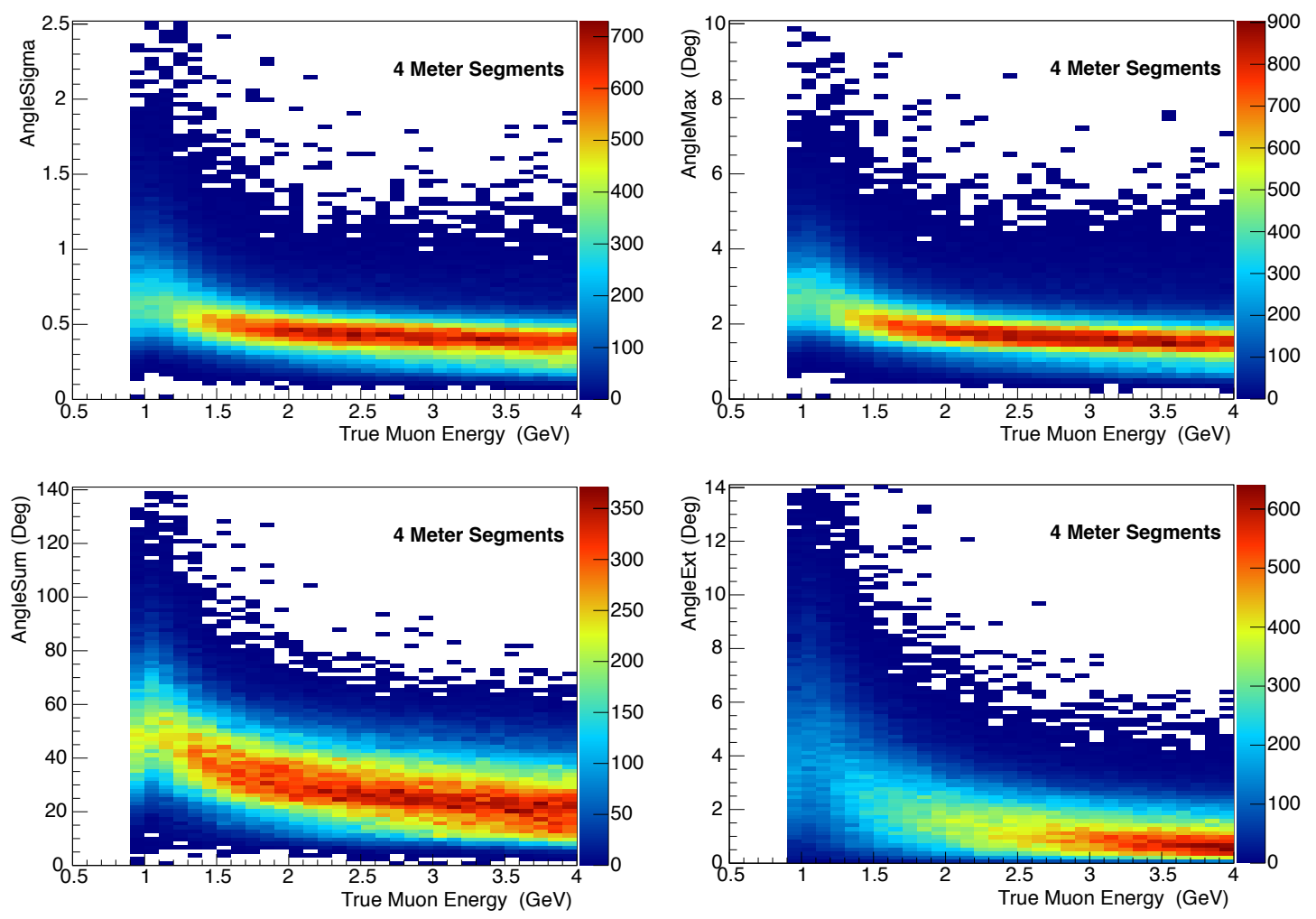

Figure 3.8: Correlation found between angleSigma (top left), angleMax (top right), angleSum(bottom left) and angleExt (bottom right) and true muon energy. These variables were calculated through 4 meter segment analysis of the muon tracks. The correlations observed on these distributions are in general agreement with the expected correlations but are noticeably different amongst one another. 


\section{Chapter 4}

\section{Muon Energy Reconstruction}

The understanding of muon multiple scattering gained through the studies discussed in Sec. 3.3.2 was utilized to develop a technique to estimate muon energies. While the correlations depicted in Fig. 3.8 are not as pronounced or as linear as the one employed by the track length method in Sec. 2.2, they clearly depict how scattering behavior differs through large energy ranges. This chapter discusses the methods through which multiple scattering information was used for muon energy reconstruction.

\subsection{Single Variable Approach Methodology}

The first approach at energy reconstruction employed an analogous technique to the one described on Sec. 2.2.1 for the track length method. A unique mathematical function was fit to each profile corresponding to the correlations shown in Fig. 3.8. Unlike the linear nature of the correlation between track length and energy, the distributions for scattering-related variables show clearly more complex behavior and, thus, more complicated functions were needed to describe them.

Figure 4.1 shows the correlation profiles with the fitted fifth-order polynomial functions which were found to best describe them. It is important to point out, however, that these 
functions are but mathematical constructs which describe the given profiles and are not a description of multiple scattering behavior alone. ${ }^{1}$ Nevertheless, their usefulness for reconstruction can be exploited and evaluated in the same manner as was done for the track length method in Sec. 2.2.1.
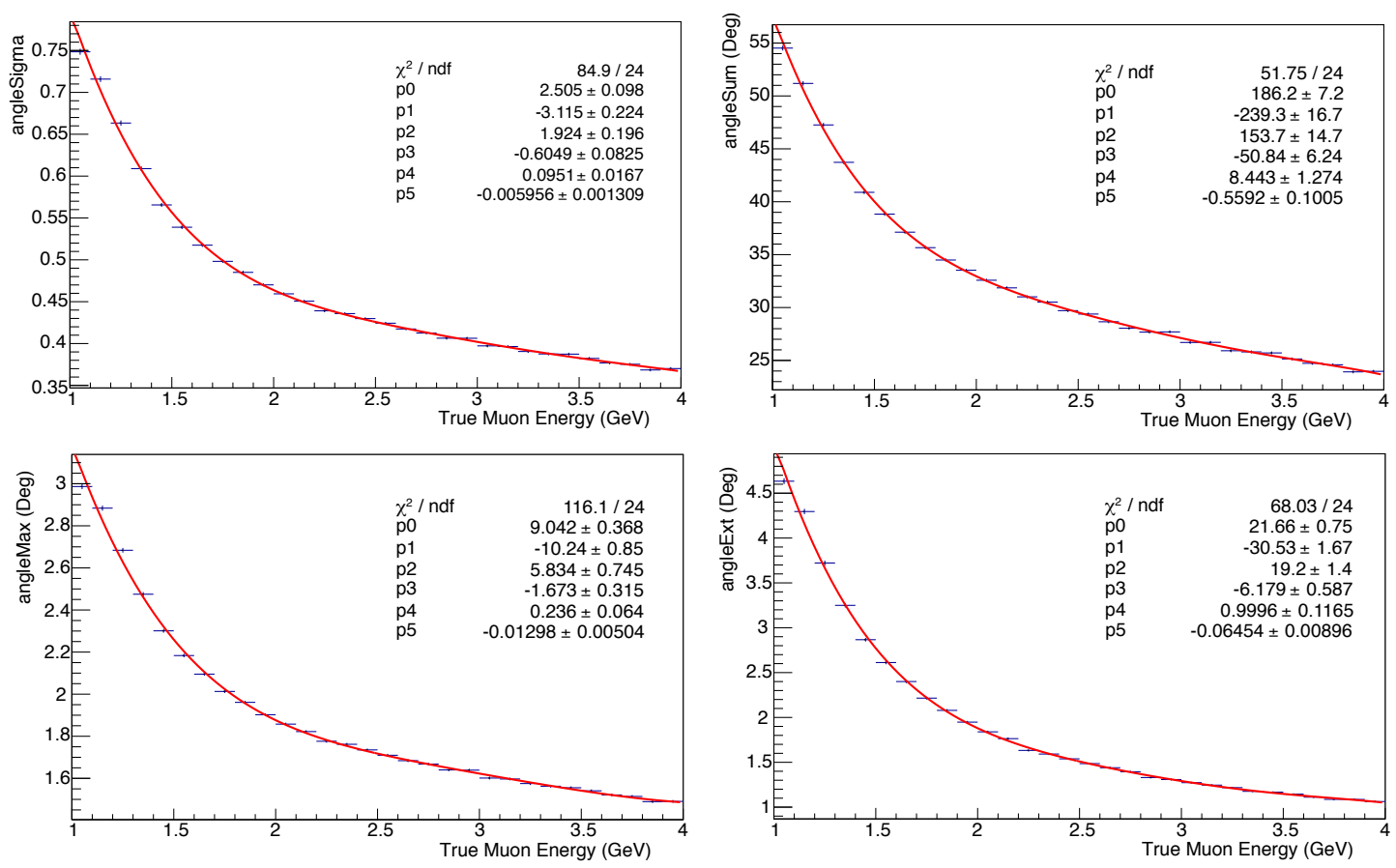

Figure 4.1: Profiles from the distributions shown in Fig. 3.8 with errors corresponding to the standard deviation for each bin. The red lines correspond to the fifth-order polynomials which best fit the profiles in the given range whose parameters are shown for each plot.

The functions fitted to the correlations of interest describe the scattering variables in terms of the true muon energy. In order to make energy estimations whose inputs are scattering variables the inverse relation is needed. While inverting the axes on the given profiles appears as an intuitively valid alternative, doing so would necessarily introduce

1 As previously discussed, the variables used in this method as gauges of multiple scattering are dependent on the particularities of the detector's geometry as well as its spatial and angular resolution. It would be incorrect to assume that the direct relation between these functions and raw scattering data is understood, as no claim is hereby made that these fifth-order polynomials describe the scattering phenomenon itself. 
an unintended assumption for the energy distribution.

Careful consideration of the calculated errors on these profile histograms reveals that while the error bars on the horizontal axis are approximately equal in magnitude, this is not the case for those on the vertical axis. The uniform energy distribution of the simulated muons (discussed in App. A.1) implies that the number of events will be equally distributed amongst all bins - minimum discrete ranges - on these histograms, for any choice of bin size within the generated energy precision. Therefore, it is both the value and the error on the vertical axis which contain scattering information that is truly independent of the energy distribution of the sample. Inverting such distributions would yield information valid specifically for muons with the given energy distribution and, thus, extrapolating it to describe any different sample of muons would require additional manipulation. ${ }^{2}$

As a consequence, a simpler method was used to avoid the problem of high-order polynomial inversion. For each of the distributions in Fig. 4.1 a table of values was constructed, containing enough entries to gauge energy ranges of at least $0.1 \mathrm{GeV}$. The reconstruction procedure entailed determining the variable of interest for a given muon track (either angleSigma, angleExt, angleMax or angleSum) and finding the energy value most closely associated with it on the table. This energy value was assigned to the muon track as its reconstructed energy.

2 The introduction of assumptions for the energy distribution of the muons is intentionally avoided in these studies. Given that the energy is the unknown parameter which we are trying to measure, any assumption of its distribution will bias the method and potentially influence the results. Assumptions for the energy distributions could be introduced in the reconstruction method with careful considerations. However, the focus of this analysis is to measure the viability of scattering-based methods alone, introducing as few external parameters as possible for their evaluation. 


\subsubsection{Single Variable Method Evaluation and Results}

This simple approach at reconstruction is certainly not the optimal use of these correlations. The "table lookup" method is not only computationally slow for large samples, it also limits the precision to within which the energy can be estimated in certain ranges. In addition, given that these high-order polynomials only describe the behavior of the variables within the given range, any input beyond the scope of the profile plots would yield values for the reconstructed energy which are meaningless, as they are not derived from an understanding of multiple scattering behavior.

Nevertheless, the value of this approach lies not in its usefulness for overall reconstruction alone. Despite its simplicity, it's capable of demonstrating whether a fruitful method for energy reconstruction can be achieved through the use of scattering information. The process of evaluating these first energy estimations yields a measure of the "usefulness" of each of these variables, which justifies the pursuit of more robust approaches at reconstruction through multiple scattering. The evaluation of this method was done through the qualitative and quantitative approaches described in Sec. 2.2.2.

The distributions in Fig. 4.2 show the results of energy reconstruction made on the generated testing muon sample by this "table lookup" method. Note that the resolution plots contain data from the labeled "reliable" regions of their corresponding qualitative evaluation plots only. 

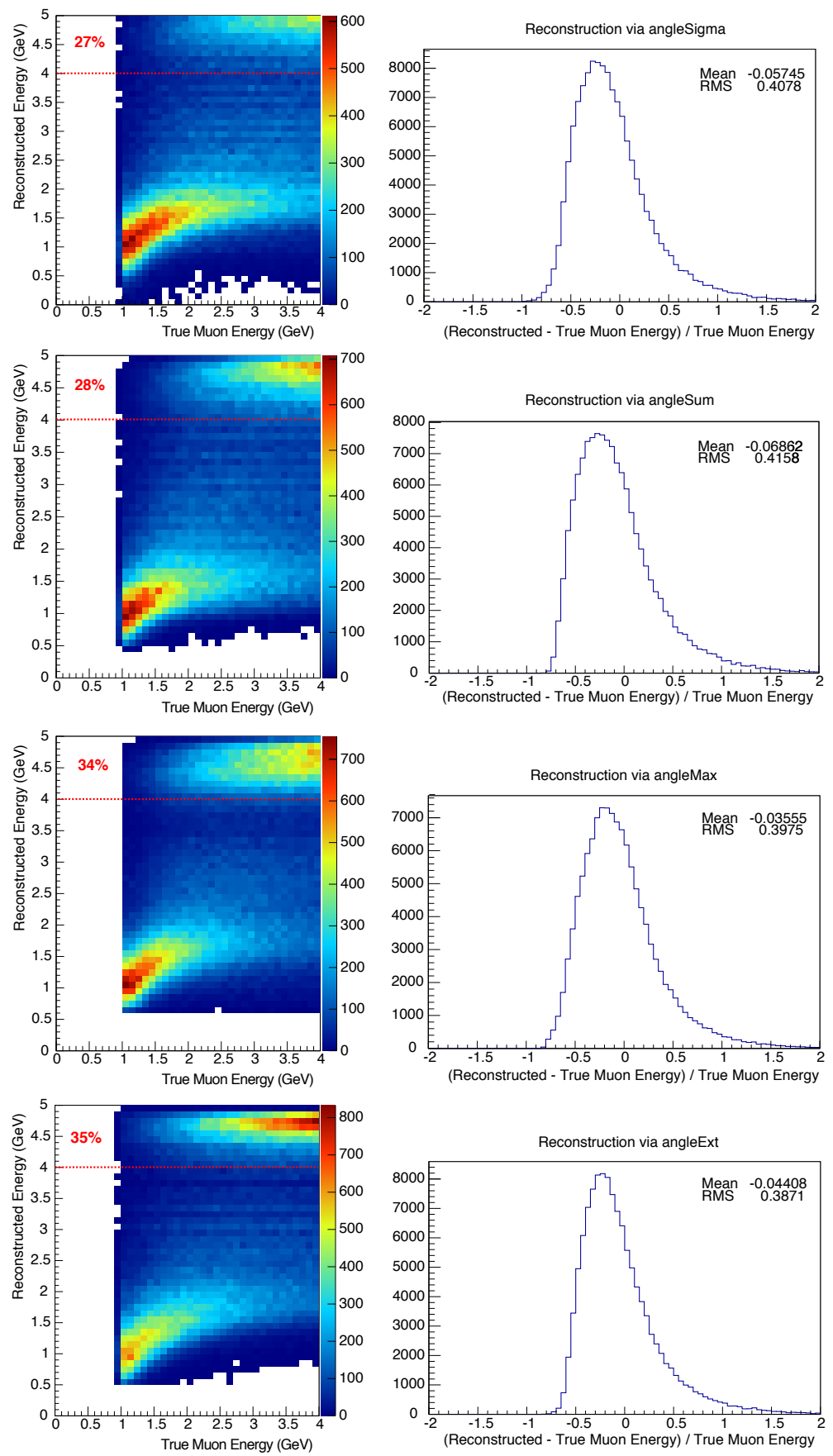

Figure 4.2: Evaluation of the "table lookup" reconstruction method. Left: Qualitative. The dotted lines signal the $4 \mathrm{GeV}$ reconstructed energy. The regions above this limit are populated with events whose input is beyond the scope of Fig. 4.1. The information from these regions is defined as "unreliable". Right: Quantitative. These distributions only contain events from the "reliable" regions of their corresponding qualitative evaluation plots. The RMS value contains information about the energy resolution. 
The most important conclusion which can be drawn from the quantitative evaluation plots in Fig. 4.2 is the fact that scattering information can, indeed, be used to make muon energy estimations. The reconstructed energy obtained by this method has a resolution which can be gauged by the root mean square and is offset by a percentage given by the mean value of its corresponding distribution.

The qualitative evaluation distributions also show clear limitations to the potential reach of the method. The labeled "unreliable" region on these plots is populated by events whose input parameter - their angle-related variable - lies beyond the scope of the reconstructing function.

When the input value for a track is smaller than those defined by the polynomial functions in the range shown in Fig. 4.1 a higher value for the energy is reconstructed. These values are associated with the function's behavior beyond the fit's scope and are, thus, arbitrary with respect to scattering behavior. Given the lower limit to the resolving power of small-angle scatterings in the geometry of the detectors, some percentage of the tracks will simply hold insufficient scattering information for this method to yield any reliable energy estimation for them. There are two consequential hypotheses which derive from this qualitative analysis:

1. The "unreliable" regions of the qualitative evaluation plots become more populated for higher values of true muon energy. Such behavior is expected given that higher energy particles undergo smaller scatterings. This behavior indicates that the reliability of multiple scattering based estimations and the true particle's energy are inversely related, which implies a threshold for energies larger than which the method cannot be employed to reconstruct.

2. The "unreliable" regions on the plots corresponding to reconstruction made with different variables are not populated with information from the same subset of events. For example, while a given muon track might have a smaller value of angleSigma 
than the method can resolve, it is possible that its value for angleExt still yields useful scattering information. Therefore, the percentage of events in a given "reliable" region might be smaller than the real fraction of events which hold useful scattering information.

The development of a multi-variate technique is naturally pertinent, not only to increase the scope of the method, but also to exploit the different traits of scattering behavior captured by each of the related variables.

\subsection{Multi-Variate Approach Methodology}

A multi-variate approach at energy reconstruction was developed with the objective of potentiating the differences captured by all scattering variables as well as increasing the percentage of events which could be impacted by it. Such a method should be based on a greater understanding of the given correlations and should attempt to overcome the limitations given by the table lookup method.

The development of this reconstruction technique was made through the use of Artificial Neural Network (ANN) algorithms, further explained in App. B. An ANN examines training sample datasets containing variables correlated to a "target" value in order to build optimized algorithms that estimate the target variable on a test sample. This process is based on self-evaluated iterations of semi-randomized sets of weights and connections between all input variables in the training sample. Through this process, it is expected that the ANN will identify complex correlations between the input values and utilize them to estimate the target value as reliably as possible.

Testing and training datasets containing true energy information and the four anglerelated variables were used as input for the $\mathrm{ANN}$ algorithm. In order to confirm the ability of the algorithm to identify the correlations exhibited by Fig. 4.1, individual parameters were used as input prior to the development of the reconstruction method. 
The details of these tests are discussed further in App. B.2. With sufficient evidence of the ANN's ability to identify such correlations, the complete datasets were given as input to the ANN, which produced an optimized energy reconstruction algorithm.

In the software environment, the energy reconstruction algorithm developed through the use of ANNs was given by a $\mathrm{C}++$ function. For any muon track, the reconstruction function is given angleSigma, angleExt, angleMax and angleSum as input and returns a value for the reconstructed energy of the muon.

\subsubsection{Multi-Variable Method Evaluation and Results}

In the process of optimizing the reconstruction algorithm, the ANN evaluates it though the use of the test sample. Thus, evaluating this method by its performance on such sample could exploit a bias of the method to perform better for that sample, if such bias existed. A third sample of events with identical parameters was used in order to evaluate this method.
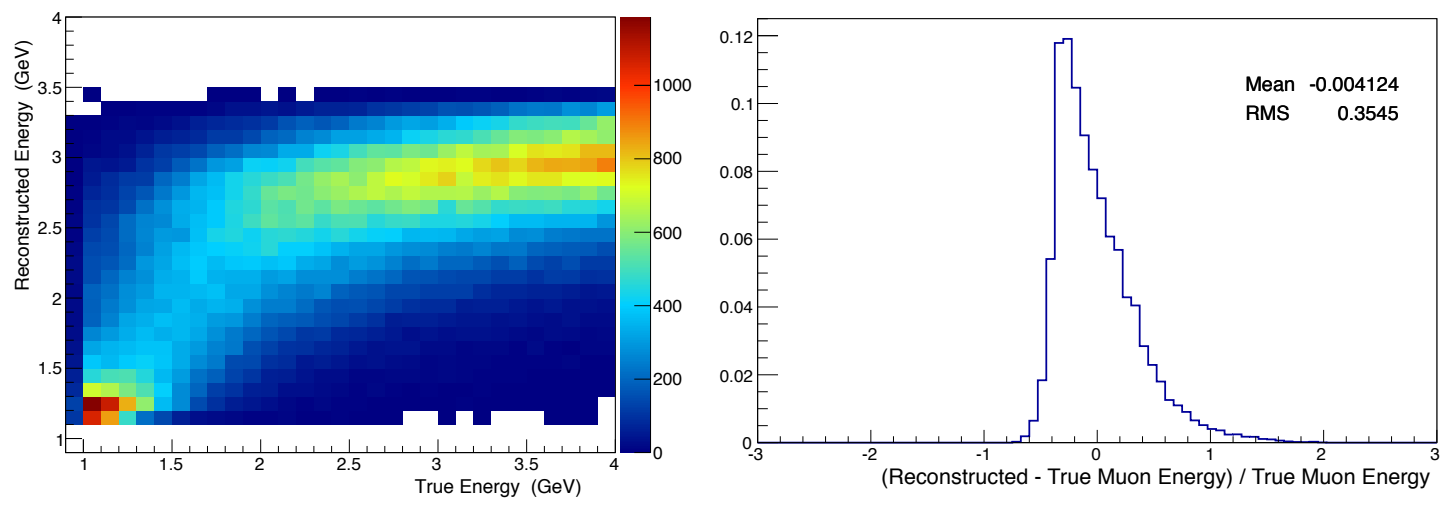

Figure 4.3: Evaluation of the reconstruction method on a single muon sample. Left: Qualitative. Shows better performance for lower true energy values. Quantitative: Shows better performance for this mutivariate approach compared to the method evaluated in Fig. 4.2.

It could be argued that the use of a sample with identical parameters for this purpose 
would not eliminate potential bias toward a sample of such characteristics. Therefore, the evaluation of the reconstruction method was done using two separate samples: This single muon simulation sample and a $\mathrm{NuMI}$ beam $\mathrm{MC}$ sample for $\mathrm{NO} \nu \mathrm{A}$. The second sample is a simulation of neutrino interactions in the NO $\nu$ A FD from which only tracks with good correspondence to muons were selected. The characteristics of this sample as well as the selection process for muon tracks within it are discussed in App. A.2.
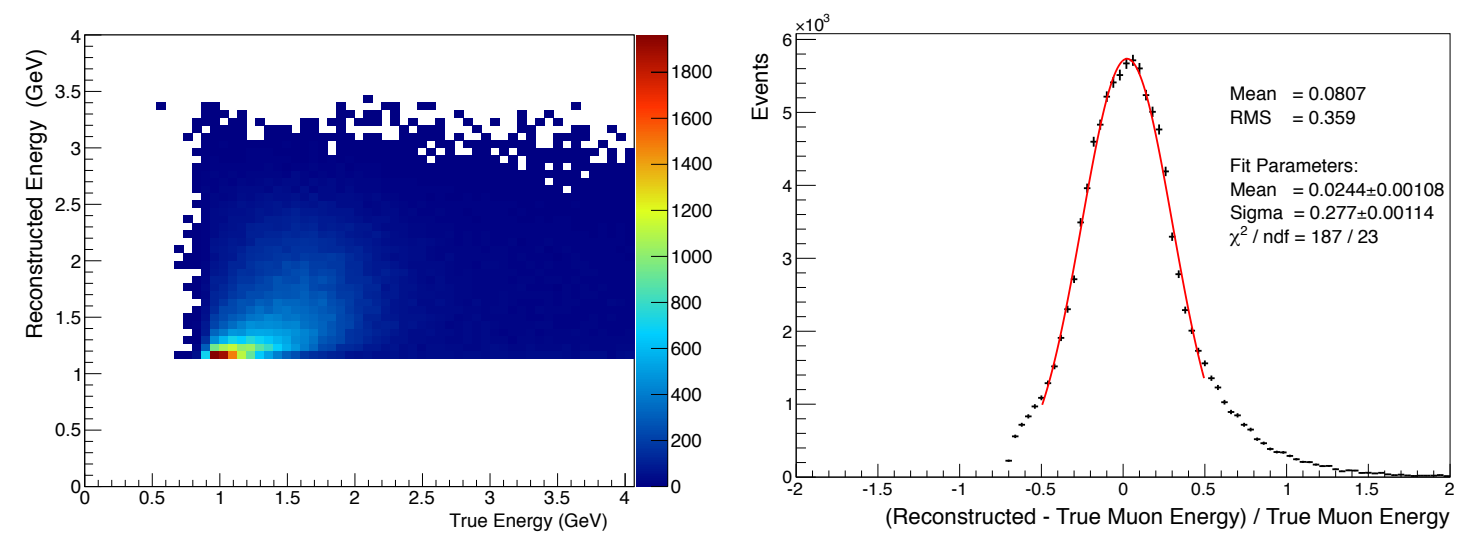

Figure 4.4: Evaluation of the reconstruction method on muons from a NuMI MC sample (App. A.2). Left: Qualitative. Shows better performance for lower true energy values. Shows an apparent discrepancy from Fig. 4.3 for higher true energy events due to the difference in energy distribution for each sample. Quantitative: Shows better performance for this muti-variate approach compared to the method evaluated in Fig. 4.2. Shows general agreement with Fig. 4.3. The red line is a gaussian fit whose parameters are shown. The energy resolution for this method is $\sim 27.7 \%$.

Figure 4.4 includes the gaussian fit whose standard deviation measures this method's energy resolution. It is important to point out that the tails of this distribution are not included for the fit given their uneven behavior, which derives from the characteristics of the sample. The seemingly unexpected behavior on the region to the left of the gaussian fit shows an abrupt decrease in value which is caused by a restriction on the true energy of the muon sample. Given that the method was developed with a training sample with true energy values up to $4 \mathrm{GeV}$, the testing was done for events with true energy within the same limits. This behavior is similarly displayed by all quantitative evaluation plots 
in this chapter given that this energy restriction was imposed to all muon samples.

It is evident from the comparison of the evaluations shown in Fig. 4.3 and 4.4 that the method's performance is consistent between samples with different energy distributions. ${ }^{3}$ Note that the less-populated region at higher energy values for the qualitative evaluation on the NuMI MC sample (Fig. 4.4 left) is a consequence of the sample's energy distribution (Fig. A.1) which is also much lower at higher energies.

\footnotetext{
${ }^{3}$ Given that the selection process for events in the second sample has less strict requirements for purity and efficiency of the muon tracks (discussed in App. A.2), exact coincidence of the method's performance in both samples is not expected.
} 


\section{Chapter 5}

\section{Discussion of Results}

In order to better understand the implications of the results accomplished by this work, it is important to relate them back to their original objective within the oscillation analyses at $\mathrm{NO} \nu \mathrm{A}$. Energy reconstruction through the multiple scattering method can be used for those events with escaping muons for which the method was developed. This reconstruction method has the potential to impact the overall analysis by improving the energy resolution of the uncontained events sample, which contributes to the results as shown in Fig. 1.2.

The contribution of the uncontained sample to the $\sin ^{2} 2 \theta_{23}$ and $\Delta m_{23}^{2}$ contours for different values of energy resolution is depicted in Fig. 5.1. It is true that the uncontained sample is not the most important subset of the events which would produce the expected results of $\mathrm{NO} \nu \mathrm{A}$ analyses. However, it can contribute by adding larger statistics to the overall dataset. In addition, during the building stages of the $\mathrm{NO} \nu \mathrm{A}$ experiment, the FD will be only partially built and escaping muons will constitute a larger fraction of the event sample. 


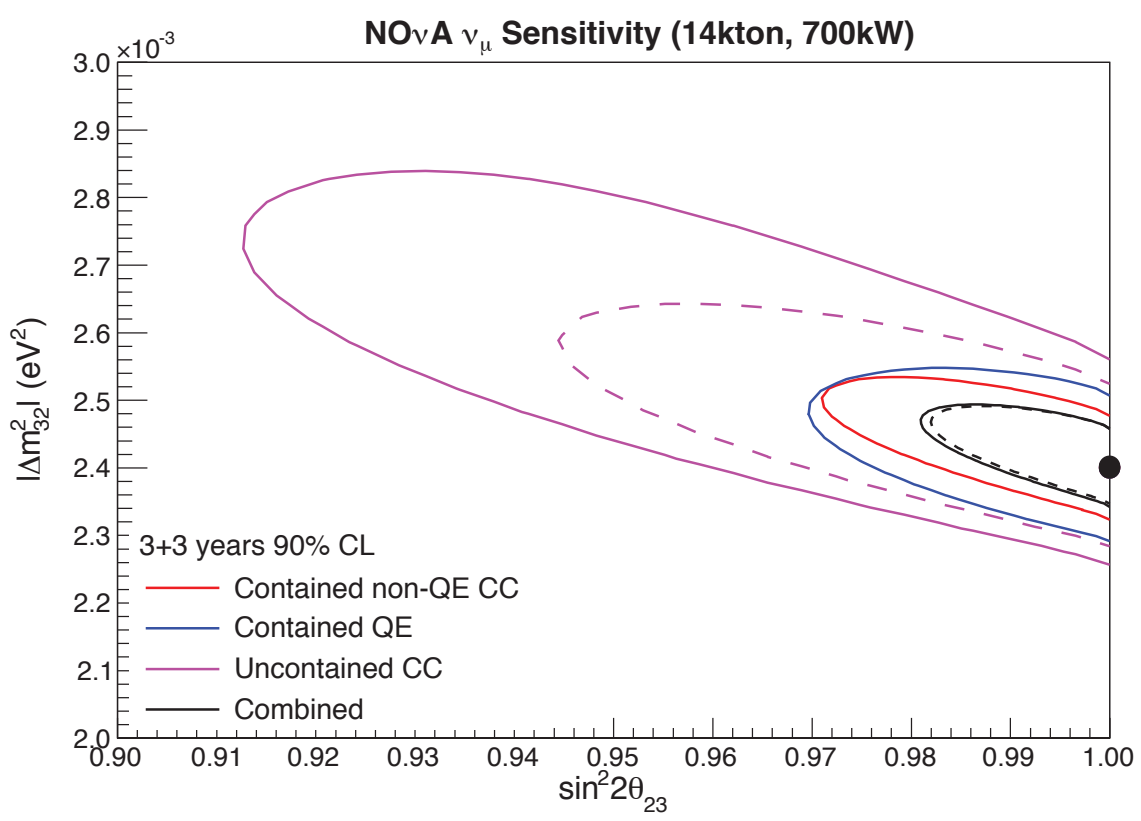

Figure 5.1: $\mathrm{NO} \nu \mathrm{A}$ expected sensitivity for different resolutions of the uncontained sample. Solid lines: The same contours shown in Fig. 1.2. The uncontained sample is not entirely reconstructed, only visible energy values are used for both the muon and hadron components. Dotted lines: The contour given by the uncontained sample if its energy resolution were improved to reach 50\% (pink) and the contribution of this change to the combined contour (black). [6]

\subsection{Statistical Impact of this Method}

The long term contribution of the method of multiple scattering for energy reconstruction can be estimated by the percentage of events which it can visibly impact. Figure 5.2 depicts such fraction, accounting for the constraints on the restrictions that the method imposes on the minimum track length and the range in true energy of the events which it can reconstruct. The expected percentage of events in the FD whose energy could be reconstructed by this method is $\sim 15 \%$, with the obtained resolution of $\sim 27.7 \%$. 


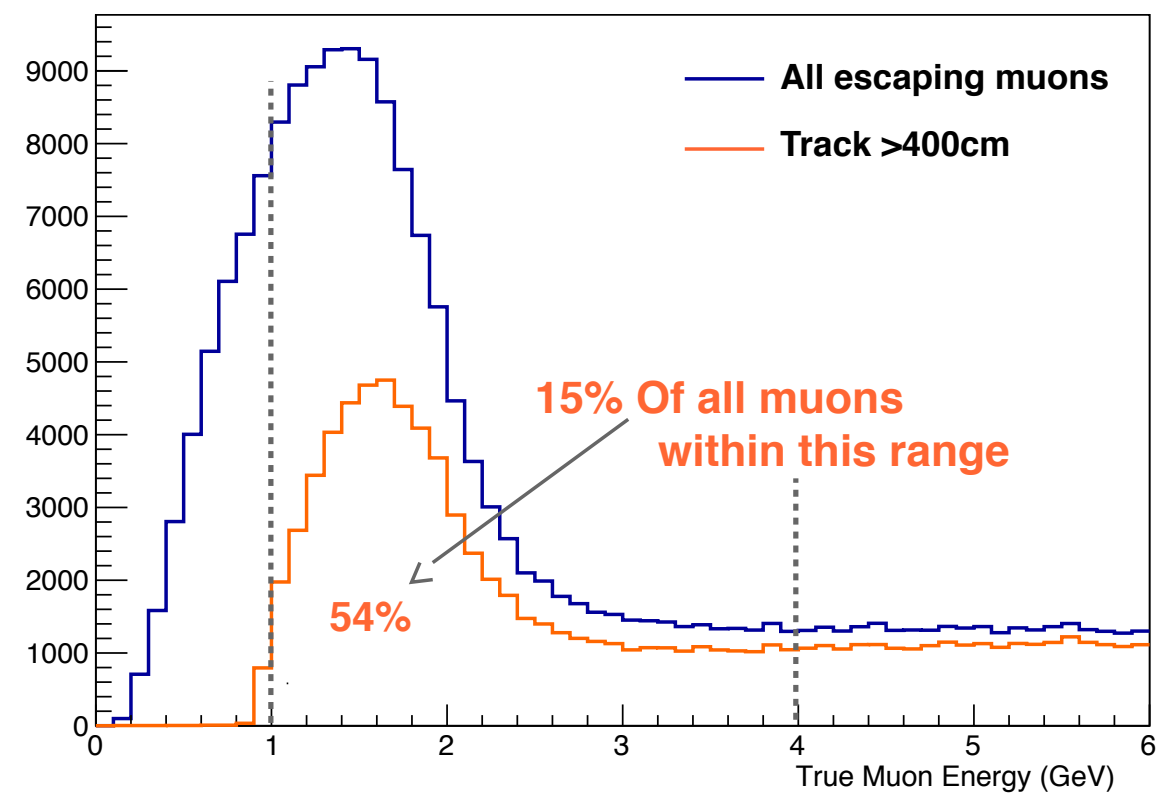

Figure 5.2: Statistical impact of the multiple scattering based reconstruction method. Blue:The true energy distribution of escaping muons from neutrino interactions within the detector, given by $\mathrm{MC}$ truth information from the NuMI beam MC. Orange: The subset of these escaping muons with tracks which are long enough to reconstruct with the segment length chosen for this method. This distribution represents $\sim 15 \%$ of all muons from neutrino interactions expected in the FD (Plot not scaled to a specific running time).

Although the results of this work are obtained through the analysis of scattering behavior for the chosen segment length of four meters, information obtained though the analysis of shorter segments could be incorporated to include a larger percentage of the data. While the objective of this work was only to study the usefulness of multiple scattering for energy reconstruction, the treatment of escaping muon events within the oscillation analysis will include a combination of approaches which optimizes the energy resolution. Some alternatives for the optimization of this method will include a combination with other parameters unrelated to scattering, such as the lower limits to the energy set by the track length and energy deposition methods. 


\subsection{Conclusion}

The phenomenon of multiple scattering of muons within the $\mathrm{NO} \nu \mathrm{A}$ detector was successfully studied in terms of its usefulness to gauge particles' energies. A technique was developed to measure muon scattering within the detector geometry and identify the correlation with the energy of multiple variables related to the phenomenon. These correlations were found and measured and further examination determined their potential for energy reconstruction.

An energy reconstruction method though the use of multiple scattering information was developed for muons in the $\mathrm{NO} \nu \mathrm{A}$ detector. The method was also evaluated on $\mathrm{MC}$ simulations and yielded a resolution of $\sim 27.7 \%$ and an expected statistical impact of $\sim 15 \%$ on the expected data in the FD. 


\section{References}

[1] M.C. Gonzalez-Garcia and Michele Maltoni. Phenomenology with Massive Neutrinos. Phys.Rept., 460:1-129, 2008, 0704.1800.

[2] NOvA Collaboration. Nova public document 8890. 2013.

[3] D.S. Ayres et al. The nova technical design report. 2007.

[4] J. Beringer et al. Review of particle physics. Phys. Rev. D, 86:010001, Jul 2012.

[5] N. Agafonova et al. Momentum measurement by the multiple Coulomb scattering method in the OPERA lead-emulsion target. New Fournal of Physics, 14(1):013026, January 2012, 1106.6211.

[6] M. Ambrosio et al. Muon energy estimate through multiple scattering with the MACRO detector. Nucl.Instrum.Meth., A492:376-386, 2002, physics/0203018.

[7] NOvA Collaboration. Nova internal document 9022. 2013.

[8] NOvA Collaboration. Nova internal document 7294. 2013.

[9] J.A. Thomas and P.L. Vahle. Neutrino Oscillations: Present Status and Future Plans. World Scientific Publishing Company Incorporated, 2008.

[10] R. Brun and F. Rademakers. Root - an object oriented data analysis framework. Nuclear Instruments and Methods in Physics Research A, 389:81-86, February 1997.

[1 1] Ziro Maki, Masami Nakagawa, and Shoichi Sakata. Remarks on the unified model of elementary particles. Prog. Theor.Phys., 28:870-880, 1962. 


\section{Appendix A}

\section{The NO $\nu$ A Event Generator and the Single Muons Sample}

The event generator is a functionality within the $\mathrm{NO} \nu \mathrm{A}$ software which produces a Monte Carlo simulation of events in the $\mathrm{NO} \nu \mathrm{A}$ detector. While the specific algorithm of the event generator is not directly relevant to the technique development described in this thesis, a rough description of its workings is necessary to describe the simulations used in the analysis.

The objective of the event generator is to simulate the behavior of particles within the detector and to produce identical output to that which the detector and reconstruction algorithms would produce when encountering such events. The algorithm simulates not only the behavior of the individual particles within the detector material, but also the performance of the detector's instrumentation and software reconstruction algorithms to their known precision and uncertainties.

The greatest advantage of analyzing the events produced by this generator is that its output preserves all the information about the simulated particles. Therefore, the performance of the detector can be measured with respect to the real scenario which would trigger specific responses. The generated particle information variables are denoted by 
the term "Monte Carlo truth".

A specific functionality of the event generator is to produce events containing information about single particles. For a given set of parameters, a single particle specified by the user will be simulated in the detector. The output of this functionality will be in the form of individual events (time windows) with only a single particle interacting in the detector, respectively.

The input parameters of the single particle event generator are shown below. This set of parameters determines the characteristics of the particles produced as well as their specific kinematics.

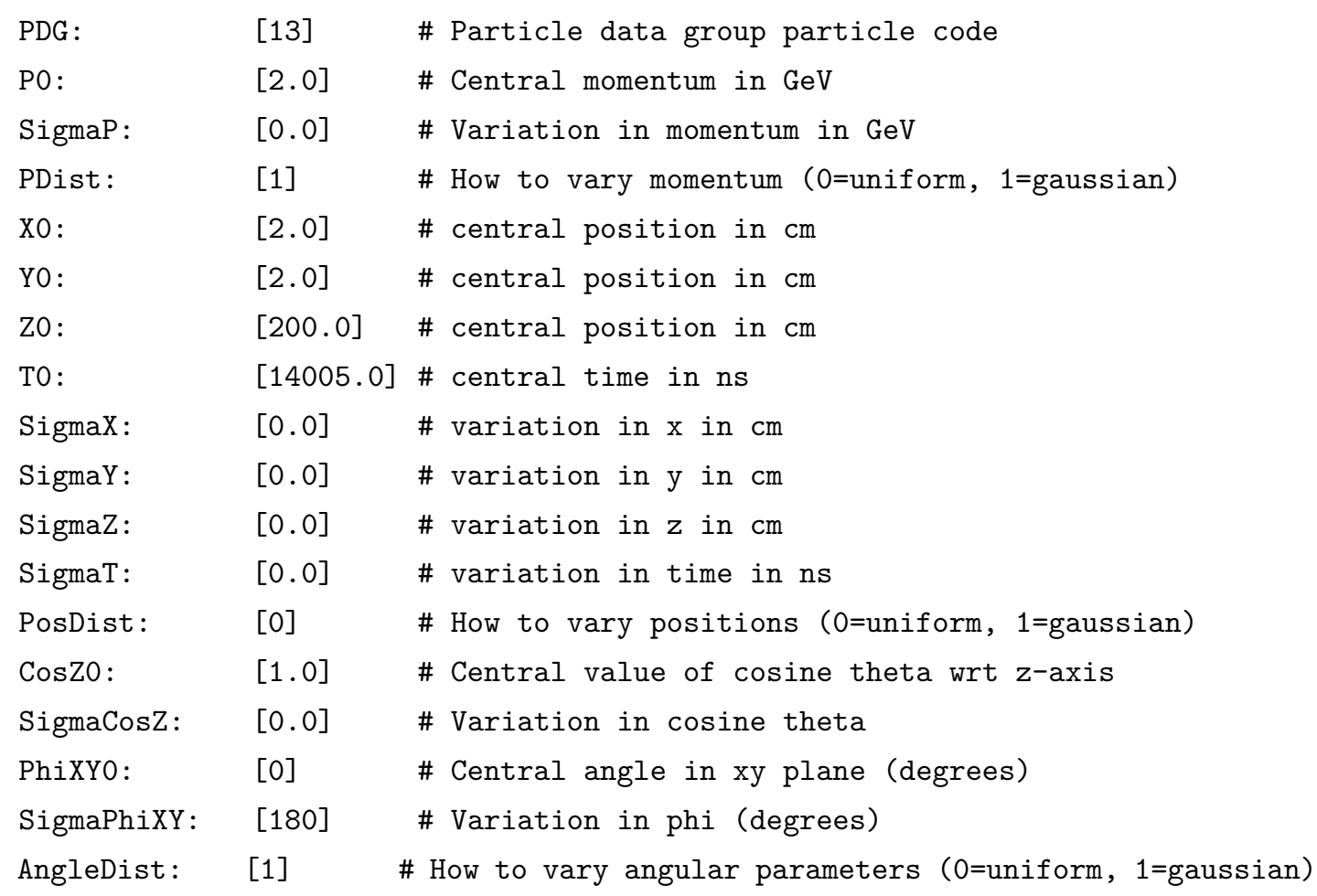




\section{A.1 Single Muon Production}

The simulation used for the studies described on chapters 3 and 4 was produced using the single particle functionality of the event generator. Given that the objective of the studies was to determine a correlation between scattering behavior and energy, the muons in the sample were required to fulfill two constraints:

1. Their kinematics and energy distribution must span all the possible values expected for neutrino daughter muons from the NuMI beam.

2. All possible parameters must be uniform across all energy values to ensure that the only correlation examined by this analysis is that of the scattering behavior.

The main parameters in question for the single production event generator were the energy distribution and the angle with respect to the detector's z-axis. For the case of the energy, the chosen parameters were based on the energy distribution of expected events from the NuMI beam given by Fig. A.1. These values were chosen to yield a uniform distribution of muon energies between 0 and $4 \mathrm{GeV}$, in order to span all events within the peak of the expected signal.

The angular parameters were determined by examination of the distribution given by Fig. A.1. The expected incident angle of muons from neutrino interactions in the far detector is mostly populated within the region from 0.7 to $1 \cos \theta_{z}$. The angular distribution of the generated muon sample was chosen to be uniform, spanning values of $\cos \theta_{z}$ from 0.8 to 1 . 

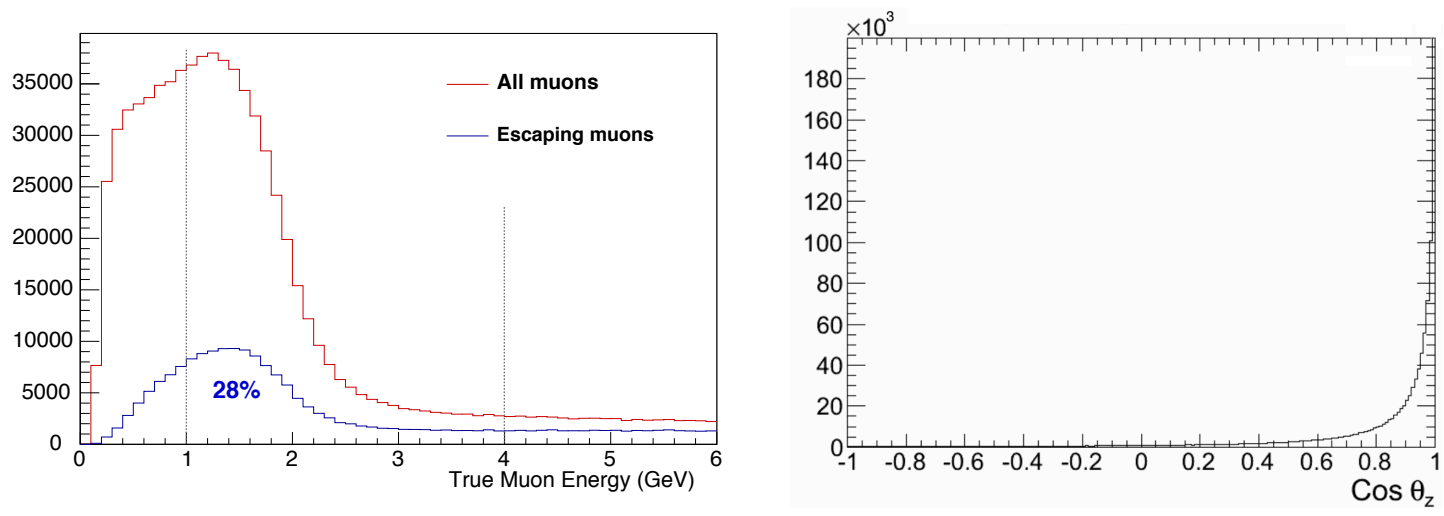

Figure A.1: Left: True muon energy distribution for all muons from neutrino interactions expected in the far detector (red) and all escaping muons (blue), which represent $\sim 28 \%$ of the expected signal within the 1 to $4 \mathrm{GeV}$ range. Right: True angular distribution for muons from neutrino interactions within the far detector. Both plots made with MC truth information from NuMI beam MC (Plots not scaled to a specific running time). [7]

Two independent samples - for independent training and testing - were generated for the studies presented in this thesis. Each sample consisted of $\sim 200,000$ events simulating single muons in the FD with uniform energy and angle distributions within the previously discussed ranges. The training sample was used for the analysis depicted in Ch.3 and Sec. 4.1 and 4.2. The reconstructions and method evaluations discussed in 4.1.1 were made using the testing sample. A third independent testing sample was also generated with $\sim 200,000$ events for the evaluations shown in Sec. 4.2.1.

\section{A.2 NuMI beam simulations for $\mathrm{NO} \nu \mathrm{A}$}

A more robust $\mathrm{MC}$ than the single particle production is used in $\mathrm{NO} \nu \mathrm{A}$ in order to simulate the interactions of NuMI beam neutrinos within the detectors. These simulations are made by the production group within the collaboration and contain information about the NuMI beam's characteristics as well as the expected neutrino cross section and the detector simulation. Each event on this sample is defined within a $500 \mu s$ time window and contains a single neutrino interaction in the case of the FD MC. 
The subset of this FD MC which is used for this analysis is a selection of events which contain muon producing $\nu_{\mu}$ interactions. Only events with reconstructed tracks of specific characteristics were selected in order for the sample to describe muon behavior with known accuracy. The following requirements were imposed for the selection of the sample used for the evaluation described in Sec. 4.2.1 and the distributions shown in Sec. 2.2.

- A muon produced in the $\nu_{\mu}$ interaction, known from MC Truth

- A reconstructed track with muon purity and efficiency greater than $80 \%{ }^{1}$

- Vertex — starting point of the track - within a fiducial volume inside the detector with borders defined $20 \mathrm{~cm}$ away from the detector's real borders

It is important to note that the purity and efficiency cuts on the sample imply that the information on the muon track is not required to be complete. However, it also describes a more realistic scenario of track reconstruction performance for muons produced in neutrino interactions.

\footnotetext{
1 Purity is defined as the fraction of hits produced by the muon which are also associated with the track. Efficiency is defined as the fraction of hits associated with the track which are also produced by the muon.
} 


\section{Appendix B}

\section{The use of Artificial Neural Network for Data Analysis}

Computational artificial neural networks (ANN) are mathematical algorithms designed to simplify complex computational problems. They do so through behavior similar to that of biological neural networks in that they consist of layers of single nodes - called neurons - which exchange information in order to produce a desired output, or solution to a problem.

The basic structure of a computational ANN is based on the concept that input is given to a set of neuron layers which will exchange it to produce the output variables. The actual ANN algorithm will perform operations to the information exchanged by particular neurons in order to produce the output. In other words, every neuron in Fig. B.1 symbolizes a continuous function (approximated by a linear combination of values) whose input are values from the previous layer and whose output is used on the subsequent layer of the algorithm. The lines in the diagram symbolize the weighing of the input by values defined uniquely for each line.

The process of the ANN used in the work presented in this thesis can be better explained by dividing it into two stages, the processing and the learning algorithms. The processing 
algorithm is depicted in Fig. B.1 and can be summarized as follows:

1. The input variables are given to the ANN algorithm.

2. Each value is weighed by a value $w_{i}$ and given as input to the first layer of neurons.

3. Each neuron computes a linear combination of its input values.

4. The output from each neuron will be used as input for the next layer of the ANN. (The process will continue for every layer previous to the output layer.)

5. The last layer will yield the output of the algorithm.

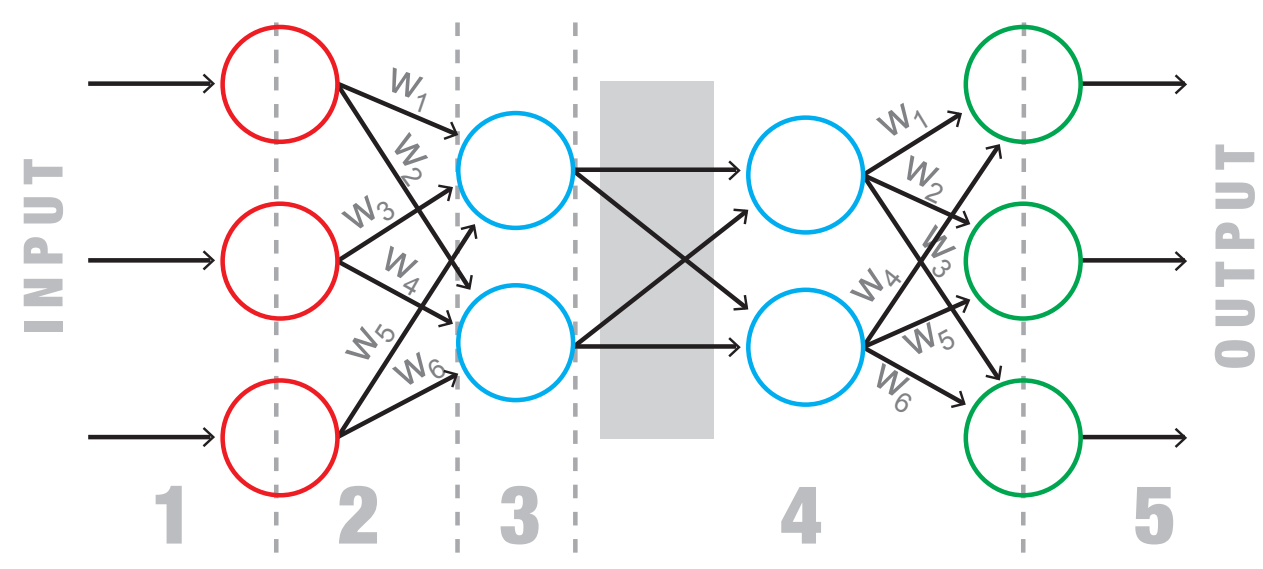

Figure B.1: ANN Functionality Diagram

A set of input values is accompanied by a set of desired output values - or target output - which the ANN is expected to produce. The learning algorithm will evaluate the ANN's performance by comparing the target output with the processing output after every iteration of the processing algorithm and it will change the weight parameters $w$ for the following iteration of the ANN process. This change in the weight parameters is made through an optimization process using semi-randomized values. This optimization process is dependent on the performance of each iteration of the method such that the weights are optimized to yield output closer to the target values at each iteration. 


\section{B.1 ROOT and the ANN functionality}

ROOT is a set of object oriented software tools and libraries which provide a framework for data handling and analysis in the $\mathrm{C}++$ language [10]. It was originally developed by GERN for particle physics data analysis and has been used for the software development of numerous particle physics experiments, including $\mathrm{NO} \nu \mathrm{A}$. The ROOT framework provides the analysis and histograming tools used for the work presented in this thesis, including the ANN functionality.

The ANN class within the ROOT framework - called TMultiLayerPerceptron - contains the previously discussed neural network functionality. It allows for user-defined parameters for the ANN such as number of epochs - procesing and learning iterations on the input - layers and neurons, as well as a choice of learning process algorithms. TMultiLayerPerceptron also provides the user with performance information useful for monitoring the behavior of the ANN.

A given set of chosen parameters must be given to the ANN function along with independent training and testing samples containing both the input and the target variables. The training functionality will execute the processing and learning algorithms on the training sample and will report the performance of the algorithm after a chosen interval of epochs in the form displayed on Fig. B.2 (left). In this way, it is possible for the user to optimize the operating parameters of the ANN based on their performance and computational time requirements.
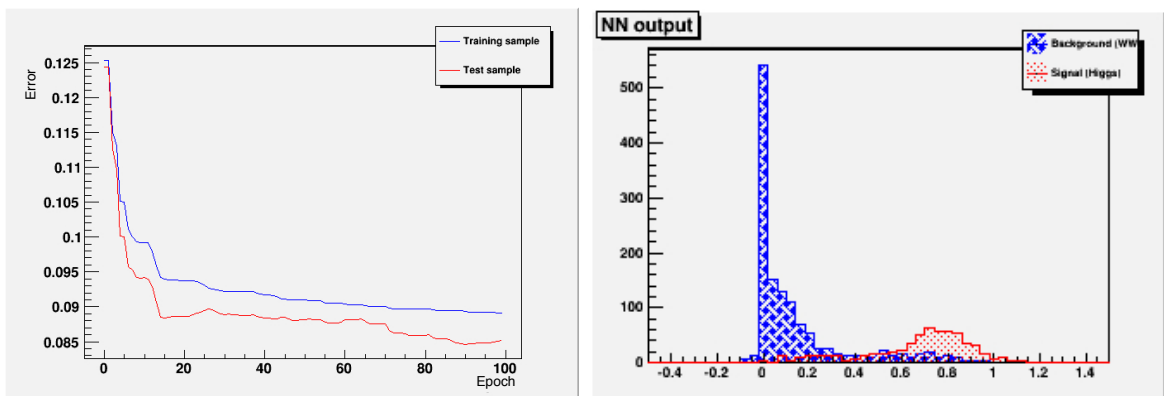

Figure B.2: ANN Monitoring Tools [10] discuss that what you want is converging behavior. 
The output of the ANN algorithm can be chosen to be a $\mathrm{C}++$ function which will mimic the behavior of the last (optimized) iteration of the process, whose outputs are the defined target variables. The number and type of target output variables can be defined by the user. A common implementation of ANN algorithms in particle physics is to distinguish expected signal and background events in a given analysis. This is done by defining the target output as a "grading" parameter such that higher "scoring" events are those with higher likelihood of being part of the signal (Fig. B.2, right).

Given that the objective of this analysis was to reconstruct a single parameter - the particle's energy - only one target parameter was defined: the reconstructed energy. The number of layers, epochs and neurons was varied and ultimately chosen to optimize the convergence of the performance on the training and testing samples (Fig. B.3) in a short computational time.The details of the input samples are discussed in App. A.1 and the testing of the output reconstruction algorithm is shown in Sec. 4.2.1
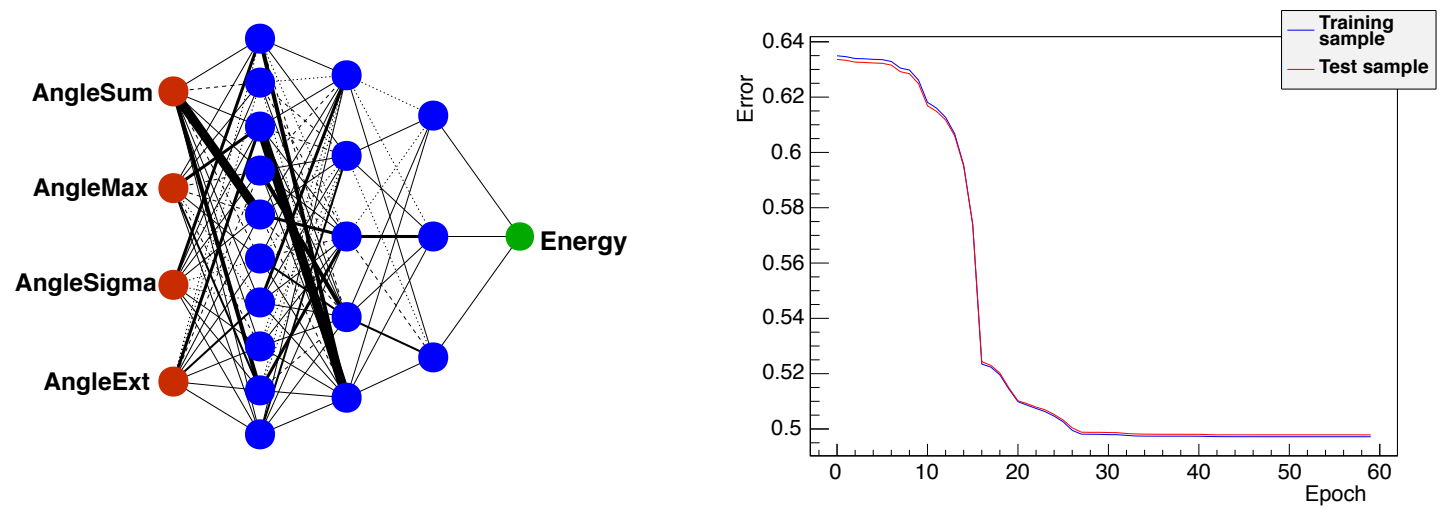

Figure B.3: Structure (left) and performance (right) of the ANN used to produce the reconstruction algorithm discussed in Sec. 4.2. Number of epochs $=60$. Neuron layer composition $=10,5,3$. Learning method $=$ stochastic optimization. 


\section{B.2 ANN testing}

Although the behavior of ANN algorithms is well understood, it is important to verify that the input variables given to it are actually correlated with the target output and that such behavior can be "understood" by the training algorithm. In order to verify the "understanding" of individual correlations between the variables used on this analysis and the true muon energy, the ANN algorithm was executed with individual input variables prior to the development of the reconstruction algorithm.

The expected result of such tests was an observable correlation between the the input variables and the output reconstructed energy analogous to the one observed between them and the true energy, depicted in Fig. 3.8. Such results are shown in Fig. B.4, which shows the expected correlations. 

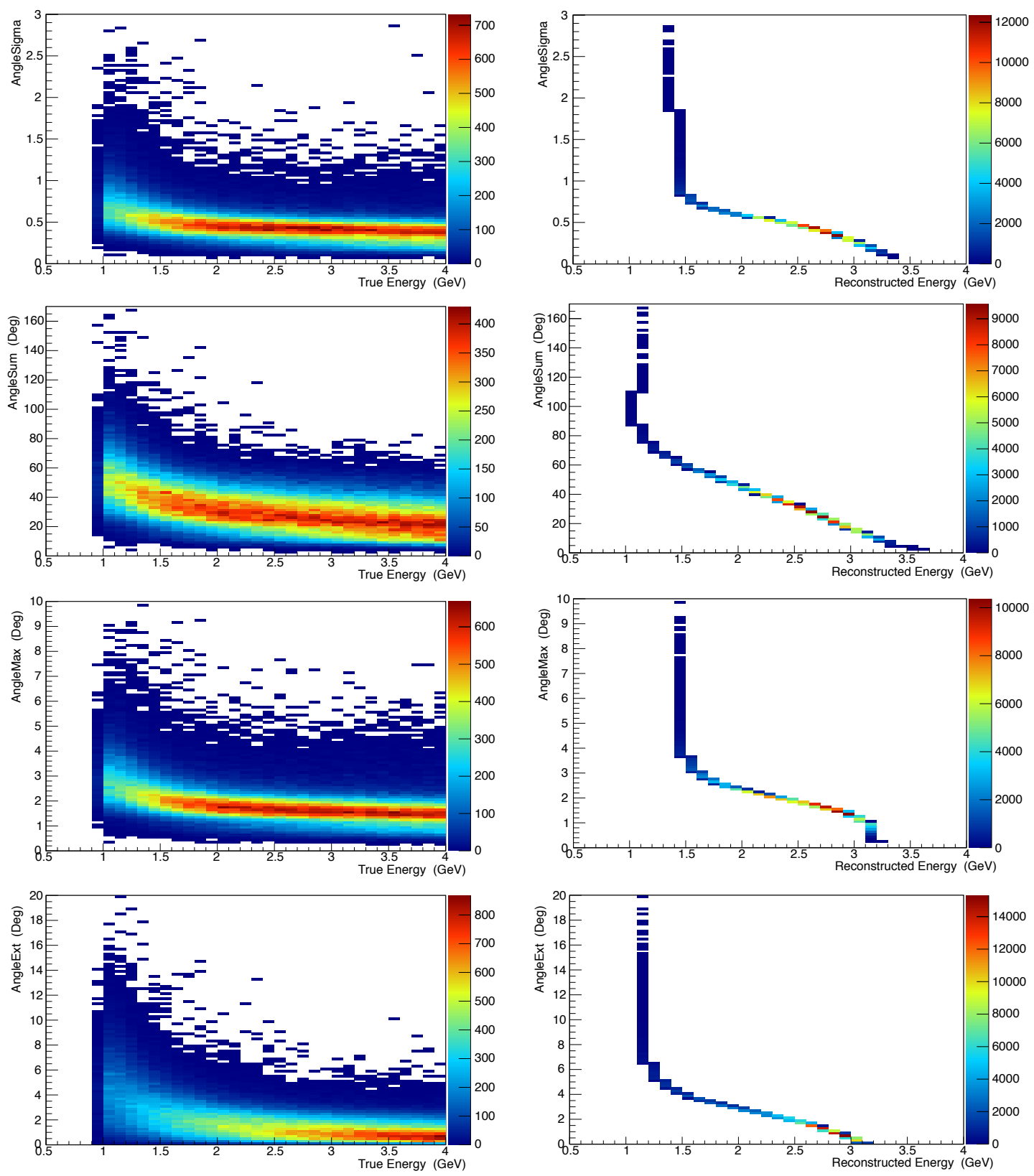

Figure B.4: Single input ANN test results. Left: Real correlations between single angle-related variables and muon true energy. Right: Correlations "understood" by the ANN. Angle variables as a function of reconstructed muon energy. Each distribution was made with data from independent ANN tests made with the single input variable on each set of plots. The shape of these distributions confirmed that the real correlation of each variable with the true energy can be "understood" and exploited by the ANN. 